\title{
Geochemistry of Sandstones from the Upper Cretaceous Sillakkudi Formation, Cauvery Basin, Southern India: Implication for Provenance
}

\author{
D. Bakkiaraj ${ }^{1}$, R. Nagendra ${ }^{1}$, R. Nagarajan ${ }^{2}$ and John S. Armstrong-Altrin ${ }^{3}$ \\ ${ }^{1}$ Department of Geology, Anna University, Chennai - 600 025, India. \\ ${ }^{2}$ Department of Applied Geology, School of Engineering and Science, Curtin University, \\ CDT 250, 98009, Miri, Sarawak, Malaysia. \\ ${ }^{3}$ Unidad Académica de Geología Marina y Ambiental, Instituto de Ciencias del Mar y Limnología, \\ Universidad Nacional Autónoma de México, México D.F., C.P. 04510. \\ Email: bakkiaraj81@gmail.com; nagarajan@curtin.edu.my; armstrong@cmarl.unam.mx
}

\begin{abstract}
Major, trace and rare earth elements (REE) composition of sandstones from the Upper Cretaceous Sillakkudi Formation, Ariyalur Group, Cauvery Basin were studied to decipher their weathering and provenance history. Texturally, these sandstones are immature, poorly sorted and grain supported. Abundance of feldspars especially, plagioclase indicates rapid deposition of sediments from a nearby source rocks. Using the geochemical classification diagram the Sillakkudi sandstones are classified as fe-sand, quartz arenite, litharenite, sub-litharenite, sub-arkose, arkose, and wacke types, which is also supported by the petrography study. The transition trace elements like $\mathrm{Co}, \mathrm{Ni}$, and $\mathrm{V}$ are higher in the Sillakkudi sandstones than upper continental crust (UCC) values. However, the Sillakkudi sandstones are lower in $\mathrm{Cr}$ (mean $\sim 21)$ content than average UCC value $(\sim 35)$. The poor correlation between $\mathrm{Cr}$ and $\mathrm{Ni}(\mathrm{r}=0.08$, number of samples $n=20)$ imply that these sandstones were derived from felsic source rocks. Similarly, the Eu/Eu* $(0.35-1.73), \mathrm{La} /$ $\mathrm{Sc}$ (1.93-9.36), Th/Sc (0.41-6.57), Th/Co (0.14-5.01), Th/Cr (0.23-2.94), and $\mathrm{Cr} / \mathrm{Th}$ (0.34-4.28) ratios support a felsic source for the Sillakkudi sandstones. The significant enrichment of $\mathrm{Zr}$, Hf, and Th in fe-sand, sub-arkose and litharenite could be related to the presence of heavy minerals, especially zircon. However, the zircon geochemistry did not affect the REE distribution and its patterns in the Sillakkudi sandstones. The Chondrite normalized REE patterns of Sillakkudi sandstones are characterized by relatively flat $\operatorname{HREE}\left(\mathrm{Gd} / \mathrm{Yb}_{\mathrm{CN}}=\sim 0.73-2.41\right.$; subscript ${ }_{\mathrm{CN}}$ refers to chondrite normalized value), enriched $\mathrm{LREE}\left(\mathrm{La} / \mathrm{Sm}_{\mathrm{CN}}=\sim 3.39-5.82\right)$ and negative Eu anomaly (mean value Eu/Eu${ }^{*}=0.80$ ). The $\mathrm{Gd} / \mathrm{Yb}_{\mathrm{CN}}$ ratios ( 0.73-2.50) are less than 2.5, which suggest that these Sillakkudi sandstones were derived from the less HREE depleted source rocks. The comparison of REE patterns and its Eu anomalies to the source rocks reveals that the Sillakkudi sandstones received a major contribution of sediments from Dharwar craton.
\end{abstract}

Keywords: Geochemistry, Zircon, sandstones, Upper Cretaceous, Sillakkudi, Cauvery Basin.

\section{INTRODUCTION}

The composition of detrital sediments is primarily controlled by the source rock composition followed by weathering and diagenetic processes to a minor extent (e.g., Armstrong-Altrin, 2009). Compositional characteristics of source rocks are generally well recorded in sedimentary products and provide valuable information about nature of source rocks and the tectonic setting of their emplacement (van de Kamp and Leake, 1995; Joo et al. 2005; ArmstrongAltrin et al. 2004; Armstrong-Altrin and Verma, 2005; Liu et al. 2007; Sinha et al. 2007; Nagarajan et al. 2007a,b; Wanas and Abdel-Maguid, 2006; Maravelis and Zelilidis, 2009). Geochemical data on sandstones of un- metamorphosed sedimentary sequences deposited in intracratonic or epicratonic basins also provide vital clues on paleoweathering conditions and variations in provenance composition and tectonics (Condie et al. 2001; Khudoley et al. 2001; Armstrong-Altrin et al. 2004; Chakrabarti et al. 2007, 2009; Carranza-Edwards et al. 2009; Araújo et al. 2010). Similarly, many studies have used the major, trace and rare earth elements (REE) geochemistry as a tool to characterize source-rock weathering, and climatic variability from the terrigenous sediments (Nesbitt and Young 1982; Cox et al. 1995; Nagarajan et al. 2007a, b; Dey et al. 2009; Ranjan and Banerjee, 2009). Thus distribution of these elements in sandstones provides clues to the geological 
processes, provenance, and tectonic settings of their respective sources (McLennan et al. 1993; Cullers et al. 1988; Umazano et al. 2009; Fanti, 2009). However, the rare earth elements contribute more to decipher the provenance than major and trace elements, since their concentration is not affected during erosion, sedimentation and diagenesis, and thus represents a homogenized average source composition (e.g. Sinha et al. 2007).

The purpose of the present study is to investigate the source rock characteristics of the Sillakkudi sandstones exposed in the Cauvery basin, southern India, using major, trace, and rare earth elements geochemistry. To identify the source rocks, the geochemical data of these sandstones are compared with granites, charnockites, and gneisses from areas located relatively close to the study area (Dharwar craton and Kerala Khondalite belt). Furthermore, the compositional changes made by the heavy minerals will be also addressed in this paper.

\section{GEOLOGICAL SETTING}

The Cretaceous succession of Cauvery basin consists of a shallow marine sequence with a very rich faunal succession of Albian - Maastrichtian age. The Cauvery basin is considered to be a rift basin (Rangaraju et al. 1993), which developed by extension during the Mesozoic break up of the Gondwana land (Prabhakar and Zutshi, 1993). Blankford (1862) carried out detailed and systematic study on this succession and divided it into three major groups as Uttatur, Trichinopoly and Ariyalur on the basis of lithology. The Ariyalur group is more widely exposed than the other two groups. Sastry et al. (1972) divided the Ariyalur group into four formations, namely Sillakkudi, Kallankurichchi, Ottakkovil and Kallamedu in upward succession. The Ariyalur Group unconformably rests over the Trichinopoly Group (Table 1). The sediments of this basin are well exposed on the coastal plain of Tamil Nadu, along the Ariyalur, Virdhachalam and Pondicherry districts. Of these, the Ariyalur area provides the most complete representation of the Mesozoic succession and afforded many works on stratigraphy, palaeontology, paleoclimate and tectonic evolution of the succession (Banerji, 1979; Ramanathan, 1979; Sundaram and Rao, 1986; Ramasamy and Banerji, 1991; Ramasamy et al. 1995; Govindan et al. 1996; Madhavaraju and Ramasamy, 1999a, b; 2001; Madhavaraju et al. 2002; Ayyasami, 2006). All the formations constituting the Ariyalur Group, except Kallamedu Formation, were deposited in the marginal marine environments (Sundaram and Rao, 1986; Madhavaraju and Ramasamy, 1999b; Madhavaraju and Lee, 2009).
Table 1. Lithostratigraphy of Ariyalur Group (modified after Sastry et al. 1972)

\begin{tabular}{|c|c|c|c|}
\hline \multirow[t]{2}{*}{ Group } & Formation & Lithology & Age \\
\hline & \multicolumn{2}{|l|}{ Niniyur } & Danian \\
\hline \multirow{4}{*}{$\begin{array}{l}\simeq \\
\triangleright \\
\sim \\
\varangle \\
\searrow \\
\simeq \\
\simeq \\
\varangle\end{array}$} & Kallamedu & $\begin{array}{l}\text { Unfossiliferous fine to } \\
\text { coarse grained sandstones } \\
\text { interbedded with siltstone, } \\
\text { sandy clay, ferruginous } \\
\text { clay and marl }\end{array}$ & \multirow{3}{*}{ Maastrichtian } \\
\hline & Ottakovil & $\begin{array}{l}\text { Fossiliferous calcareous } \\
\text { sandstone interbedded } \\
\text { with sandy clay }\end{array}$ & \\
\hline & Kallankurichchi & $\begin{array}{l}\text { Fossiliferous calcareous } \\
\text { conglomeratic sandstone } \\
\text { interbedded with sandy } \\
\text { clay, sandy fossiliferous } \\
\text { limesone, fossiliferous } \\
\text { limestone and marl }\end{array}$ & \\
\hline & Sillakkudi & $\begin{array}{l}\text { Unfossiliferous calca- } \\
\text { reous sandstone, Fossili- } \\
\text { ferous calcareous gritty } \\
\text { sandstone, Fossiliferous } \\
\text { calcareous andstones } \\
\text { interbedded with sandy } \\
\text { clay and thin band of } \\
\text { sandy limestone }\end{array}$ & Campanian \\
\hline $\begin{array}{l}\text { Trichi- } \\
\text { nopoly }\end{array}$ & & & $\begin{array}{l}\text { Late } \\
\text { Turonian to } \\
\text { Santonian }\end{array}$ \\
\hline
\end{tabular}

The Sillakkudi Formation at the base of the Ariyalur Group has been selected for this study (Fig.1). It overlies the Trichinopoly Group unconformably (Ayyasamy, 2006). This formation comprises of unfossiliferous calcareous sandstone, fossilifereous calcareous gritty sandstone, fossiliferous calcareous sandstone and interbedded arenaceous limestone with sandy clay. The basal unit of the Sillakkudi Formation consists of unfossiliferous calcareous sandstone, which is thin in the northern part of the study area, whereas it is well developed in the central and southern parts. A thick band of oolitic ironstone, sandwiched between the fossiliferous calcareous sandstone and arenaceous limestone has been reported from well sections (Madhavarju, 1996). Major part of this Sillakkudi Formation consists of sandstone and thus this study is concentrated on sandstones of this formation. The Kallankurichchi Formation unconformably overlies this Sillakkudi Formation. The Sillakkudi Formation (Fig.1) is well exposed in Mettol railway cutting (lat. $11^{\circ} 04^{\prime} 54.9^{\prime \prime} \mathrm{N}$ and long. $79^{\circ} 02^{\prime} 3.1^{\prime \prime E}$ ), Nochikkulam (lat. $11^{\circ} 07^{\prime} 55.8^{\prime \prime} \mathrm{N}$ and long. $79^{\circ} 03^{\prime} 01^{\prime \prime} \mathrm{E}$ ), and Vayalpadi (lat. $11^{\circ} 20 ' 2.5^{\prime \prime} \mathrm{N}$ and long. $\left.79^{\circ} 07^{\prime} 9.1 " \mathrm{E}\right)$. 


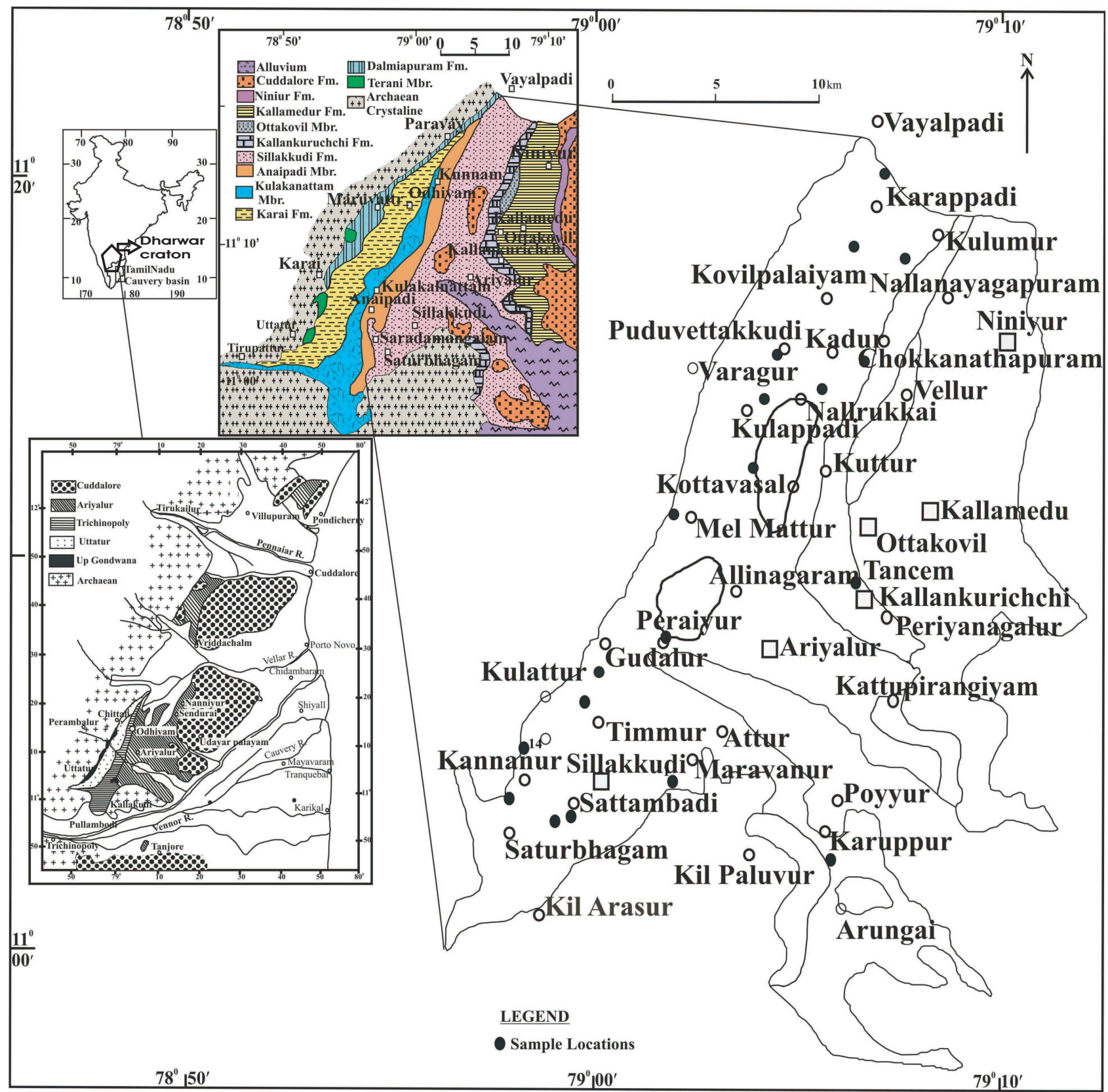

Fig.1. Geology and location map of the study area (modified after Sundaram et al. 2001).

\section{MATERIALS AND METHODS}

Thirty fresh, least weathered sandstone samples were chosen from the Sillakkudi Formation and they were washed thoroughly in distilled water to remove contamination. A detailed petrographic study was carried out for about twenty thin sections. For modal analysis, a minimum of three hundred framework grains were counted from each thin section, except matrix and cement. The point counts were done using Gazzi-Dickinson (Gazzi, 1966, Dickinson, 1970) and traditional methods. The samples were then analyzed for their major element geochemistry. The samples were digested using $\mathrm{NaOH}$ solution (solution $\mathrm{A}$ ) and are analyzed for the major elements $\mathrm{SiO}_{2}$ and $\mathrm{Al}_{2} \mathrm{O}_{3}$. Later, the samples were digested in a $\mathrm{HNO}_{3}+\mathrm{HF}+\mathrm{HClO}_{4}$ solution and were analyzed for other major elements $\left(\mathrm{Fe}_{2} \mathrm{O}_{3}, \mathrm{CaO}, \mathrm{MgO}, \mathrm{Na}_{2} \mathrm{O}\right.$, $\mathrm{K}_{2} \mathrm{O}$ and $\mathrm{P}_{2} \mathrm{O}_{5}$ ). Twenty of them were analyzed for trace and rare earth elements by Inductively Coupled PlasmaMaas Spectrometry (ICP-MS-ELAN). $0.05 \mathrm{~g}$ of each of the samples was digested with mixture of $7 \mathrm{ml}$ of $\mathrm{HF}, 3 \mathrm{ml}$ of $\mathrm{HNO}_{3}$ and $1 \mathrm{ml}$ of $\mathrm{HClO}_{4}$ for 24 hours in a tightly closed 
Teflon vessel on a hot plate at temperature $<150^{\circ} \mathrm{C}$. Later, the solution was evaporated to dryness and extracted with $10 \mathrm{ml}$ of $1 \% \mathrm{HNO}_{3}$. An internationally recognized standard reference material (SRMs) viz., GSR 4 was used for reference. Replicate analyses of samples indicate that error for major elements is better than $1 \%$, whereas the precision for other elements varies between 1 and 9\%. Based on these standards, the accuracy and precision of the analysis were within $\pm 2 \%$ for the elements like $\mathrm{Ni}, \mathrm{Cu}, \mathrm{Rb}, \mathrm{Sr}, \mathrm{Y}, \mathrm{Nb}, \mathrm{Cs}$, $\mathrm{Ba}, \mathrm{Ta}, \mathrm{Pb}, \mathrm{Th}, \mathrm{U}, \mathrm{Eu}, \mathrm{Gd}, \mathrm{Tb}, \mathrm{Dy}, \mathrm{Ho}, \mathrm{Yb}$ and $\mathrm{Lu} ; \pm 5 \%$ for $\mathrm{Cr}, \mathrm{Co}, \mathrm{Zn}, \mathrm{Ga}, \mathrm{Zr}$ and $\mathrm{Er}$; and $\pm 10 \%$ for Sc, V, Sm, and Tm. The REE data were normalized relative to the Chondrite values (Taylor and McLennan, 1985). In order to quantify the degree of weathering, the chemical Index of Alteration (CIA) was used (Nesbitt and Young, 1982) and this index was calculated using the molecular proportions as shown in the equation below:

$$
\mathrm{CIA}=\left[\mathrm{Al}_{2} \mathrm{O}_{3} /\left(\mathrm{Al}_{2} \mathrm{O}_{3}+\mathrm{CaO}^{*}+\mathrm{Na}_{2} \mathrm{O}+\mathrm{K}_{2} \mathrm{O}\right)\right] \times 100
$$

where, $\mathrm{CaO}^{*}$ is the amount of $\mathrm{CaO}$ incorporated in the silicate fraction of the rock. Correction for $\mathrm{CaO}$ from carbonate contribution was not done in this study due to the absence of $\mathrm{CO}_{2}$ value. Thus, to compute for $\mathrm{CaO}^{*}$ from the silicate fraction, the assumption proposed by Bock et al. (1998) was adopted. In this regard, $\mathrm{CaO}$ values were accepted only if $\mathrm{CaO}<\mathrm{Na}_{2} \mathrm{O}$; consequently, when $\mathrm{CaO}>$ $\mathrm{Na}_{2} \mathrm{O}$, it was assumed that the concentration of $\mathrm{CaO}$ equals to that of $\mathrm{Na}_{2} \mathrm{O}$. This procedure provides measure of the ratio of the secondary aluminous mineral to feldspar, and forms a basis for the measure of intensity of weathering.

\section{RESULTS}

\section{Petrography}

Most of the sandstones from the Sillakkudi Formation show grain supported fabric (sub-angular to sub-rounded). Generally, these sandstones consist of quartz, feldspar, rock fragments, biotite, glauconite, heavy minerals, opaque minerals and calcite cement. The main framework grains are non undulatory monocrystalline quartz, undulatory monocrystalline quartz, polycrystalline quartz, plagioclase feldspar, K-feldspar, and rock fragments. Quartz mostly in the form of monocrystalline grains, constitute on average, $76.64 \%$ of the rock volume. Some of the quartz grains show multiple deformation fractures. In polycrystalline quartz, the 2-3 crystal unit per grain variety is dominant than the $>3$ crystal unit per grain variety and some of the grains rarely shows up to 5 crystal unit per grain. Mostly quartz grains show a straight extinction and some of them show undulose extinction. Quartz with abundant vacuoles is present in many samples. The feldspars are orthoclase, plagioclase, microcline, and some perthite. K-feldspar and plagioclase are present in varying proportions among the samples and feldspars are significantly altered on the boundary and inner part of the grain. Quartz - feldspar intergrowth and perthite is also seen in some of the samples. On average, feldspar constitutes about $16.94 \%$. Lithic fragments are comparatively less abundant, which are mostly sedimentary and some are metamorphic. These rock fragments are clearly seen in the litharenite type with an overall average of about $6.13 \%$. Mica, particularly biotite, is more abundant than white mica in some samples. These sandstones contain substantial amount of calcite both as shell fragments and as calcite cement. Heavy minerals such as zircon and garnet, and more opaque minerals (magnetite and hematite) were observed. Glauconites are common in some sandstones. Calcite cement is present both in the form of micrite and spar calcite. The calcite cement constitutes 15 to $25 \%$ and matrix constitutes 5 to $15 \%$. Early formed calcite cement is masked by iron oxide materials and coated over the framework grains.

\section{Geochemistry}

The major (wt\%), trace (ppm) and rare earth element (ppm) concentrations of the Sillakkudi sandstones are reported in the Table 2. The major element compositions of the Sillakkudi sandstones are quite variable. Using the geochemical classification diagram (Herron, 1988) the Sillakkudi sandstones are classified as different rock types (Fig.2). Slight enrichment in $\mathrm{SiO}_{2}$ (wt \%) content is observed in fe-sand (the mean with one standard deviation value being $75.71 \pm 14.43 ; \mathrm{n}=8)$, quartz arenite $(74.68 \pm 9.26 ; \mathrm{n}=6)$, and sub-arkose $(71.48 \pm 4.86 ; \mathrm{n}=7)$ as compared to litharenite $(66.00 \pm 3.13 ; \mathrm{n}=4)$, arkose $(62.28 \pm 0.65 ; \mathrm{n}=$ $2)$, wacke (61.27 $\pm 0.96 ; n=2)$, and sub-litharenite (68.45), which can be attributed to the variation in quartz content among these sandstone types. $\mathrm{Al}_{2} \mathrm{O}_{3}$ content is relatively high in wacke (10.32 \pm 0.53$)$, arkose $(9.75 \pm 0.19)$, and litharenite $(7.50 \pm 1.94)$, but low in fe-sand $(4.03 \pm 1.55)$, sub-arkose $(3.10 \pm 1.25)$, and quartz arenite $(1.10 \pm 0.27)$. The $\mathrm{K}_{2} \mathrm{O}$ content is higher in arkose and sub-arkose (3.24 \pm 0.30 and $3.24 \pm 0.70$, respectively) than other rock types. This variation in $\mathrm{K}_{2} \mathrm{O}$ content is probably due to the differences in K-feldspar content among these sandstone types. Similarly, the $\mathrm{Na}_{2} \mathrm{O}$ content is higher in arkose $(2.73 \pm 0.02)$ than litharenite $(1.50 \pm 0.64)$, sub-arkose $(1.28 \pm 1.01)$, wacke $(1.16 \pm 0.17)$, and quartz arenite $(1.12 \pm 1.36)$. Low $\mathrm{Na}_{2} \mathrm{O}$ content is recorded in fe-sand $(0.21 \pm 0.29)$. The wide variation in $\mathrm{K}_{2} \mathrm{O} / \mathrm{Na}_{2} \mathrm{O}$ ratio $(0.44$ - 34.3) may be due to the low and erratic concentrations of $\mathrm{Na}_{2} \mathrm{O} . \mathrm{K}_{2} \mathrm{O}$ and $\mathrm{Na}_{2} \mathrm{O}$ 
Table 2. Major (wt \%), trace (ppm), and rare earth element (ppm) concentrations of Sillakkudi sandstones

\begin{tabular}{|c|c|c|c|c|c|c|c|c|c|c|c|c|c|c|}
\hline \multirow{2}{*}{$\begin{array}{l}\text { Rock type } \\
\text { Sample \# }\end{array}$} & \multicolumn{8}{|c|}{ Fe Sand } & \multicolumn{6}{|c|}{ Quartz arenites } \\
\hline & Sk-III & SK-IV & SK-V & PR-II & PR-II.1 & PR-III & PR-IV & PR-V & SK-II & SK-VI & Sk-XI & PR-I & PV & KP \\
\hline $\mathrm{SiO}_{2}$ & 60.41 & 55.01 & 60.57 & 84.06 & 88.27 & 89.93 & 81.59 & 85.81 & 73.71 & 75.61 & 71.15 & 90.46 & 75.34 & 61.82 \\
\hline $\mathrm{TiO}_{2}$ & 1.15 & 0.92 & 1.16 & 1.41 & 1.17 & 1.19 & 1.00 & 1.01 & 1.61 & 1.07 & 1.56 & 1.32 & 1.07 & 0.47 \\
\hline $\mathrm{Al}_{2} \mathrm{O}_{3}$ & 2.59 & 4.67 & 1.81 & 5.26 & 5.44 & 6.12 & 3.08 & 3.25 & 1.44 & 1.10 & 0.81 & 1.24 & 0.76 & 1.24 \\
\hline $\mathrm{Fe}_{2} \mathrm{O}_{3}$ & 14.93 & 14.91 & 14.40 & 7.84 & 3.48 & 1.28 & 12.04 & 8.90 & 0.08 & 0.12 & 0.76 & 4.98 & 2.62 & 2.88 \\
\hline $\mathrm{MnO}$ & 0.006 & 0.031 & 0.045 & 0.004 & 0.004 & 0.004 & 0.004 & 0.007 & 0.031 & 0.013 & 0.818 & 0.004 & 0.601 & 0.207 \\
\hline $\mathrm{MgO}$ & 0.22 & 0.20 & 0.26 & 0.03 & 0.02 & 0.02 & 0.02 & 0.01 & 0.19 & 0.19 & 0.25 & 0.04 & 0.29 & 0.27 \\
\hline $\mathrm{CaO}$ & 13.40 & 14.10 & 13.10 & 0.86 & 0.98 & 0.80 & 0.78 & 0.70 & 11.90 & 13.50 & 11.50 & 0.70 & 8.65 & 18.05 \\
\hline $\mathrm{Na}_{2} \mathrm{O}$ & 0.31 & 0.72 & 0.55 & 0.01 & 0.04 & 0.01 & 0.02 & 0.01 & 0.48 & 0.53 & 0.75 & 0.04 & 3.80 & 1.16 \\
\hline $\mathrm{K}_{2} \mathrm{O}$ & 2.61 & 2.13 & 2.60 & 0.02 & 0.06 & 0.23 & 0.10 & 0.03 & 2.18 & 1.98 & 2.78 & 0.12 & 1.97 & 3.99 \\
\hline $\mathrm{P}_{2} \mathrm{O}_{5}$ & 0.32 & 0.30 & 0.33 & 0.11 & 0.11 & 0.11 & 0.17 & 0.11 & 0.28 & 0.27 & 0.29 & 0.11 & 0.13 & 0.17 \\
\hline LOI & 4.29 & 6.81 & 6.10 & 0.35 & 0.85 & 0.43 & 0.49 & 0.19 & 8.45 & 6.54 & 8.83 & 0.87 & 5.11 & 10.03 \\
\hline Total & 100.23 & 99.80 & 100.93 & 99.95 & 100.43 & 100.12 & 99.29 & 100.03 & 100.34 & 100.93 & 99.49 & 99.88 & 100.33 & 100.28 \\
\hline $\mathrm{Sc}$ & - & 7.08 & & 8.88 & 10.52 & 12.23 & - & - & 14.71 & - & - & - & 15.32 & 4.90 \\
\hline V & - & 75.15 & & 83.64 & 94.95 & 134.97 & - & - & 92.65 & - & - & - & 91.11 & 39.65 \\
\hline $\mathrm{Cr}$ & - & 16.44 & & 17.93 & 29.31 & 22.46 & - & - & 19.52 & - & - & - & 19.03 & 9.87 \\
\hline Co & - & 8.27 & & 10.62 & 5.87 & 11.84 & - & - & 15.1 & - & - & - & 14.98 & 7.15 \\
\hline $\mathrm{Ni}$ & - & 25.73 & & 29.31 & 22.61 & 29.22 & - & - & 31.48 & - & - & - & 32.32 & 30.27 \\
\hline $\mathrm{Cu}$ & - & 27.09 & & 29.64 & 26.09 & 30.56 & - & - & 21.56 & - & - & - & 22.16 & 22.91 \\
\hline $\mathrm{Zn}$ & - & 33.79 & & 50.72 & 41.84 & 57.96 & - & - & 61.89 & - & - & - & 63.28 & 21.06 \\
\hline $\mathrm{Ga}$ & - & 18.20 & & 20.17 & 18.13 & 21.50 & - & - & 16.7 & - & - & - & 16.90 & 5.47 \\
\hline $\mathrm{Rb}$ & - & 81.43 & & 14.91 & 16.47 & 28.38 & - & - & 100.87 & - & - & - & 102.72 & 20.71 \\
\hline $\mathrm{Sr}$ & - & 318.52 & & 29.93 & 26.03 & 35.98 & - & - & 406.47 & - & - & - & 405.93 & 1170.80 \\
\hline $\mathrm{Y}$ & - & 32.89 & & 33.00 & 20.26 & 16.30 & - & - & 92.8 & - & - & - & 94.64 & 12.42 \\
\hline $\mathrm{Zr}$ & - & 2981.73 & & 3312.93 & 2991.59 & 1084.17 & - & - & 404.11 & - & - & - & 403.28 & 93.18 \\
\hline $\mathrm{Nb}$ & - & 11.25 & & 17.32 & 31.10 & 42.60 & - & - & 13.98 & - & - & - & 14.13 & 3.74 \\
\hline Cs & - & 2.00 & & 1.48 & 1.59 & 2.41 & - & - & 2.42 & - & - & - & 2.39 & 0.80 \\
\hline $\mathrm{Ba}$ & - & 2114.54 & & 198.32 & 111.08 & 221.10 & - & - & 2971.7 & - & - & - & 2988.19 & 842.07 \\
\hline $\mathrm{Hf}$ & - & 86.28 & & 93.60 & 85.54 & 154.77 & - & - & 12.5 & - & - & - & 12.45 & 2.82 \\
\hline $\mathrm{Ta}$ & - & 0.91 & & 1.01 & 1.81 & 2.40 & - & - & 0.69 & - & - & - & 0.75 & 0.23 \\
\hline $\mathrm{Pb}$ & - & 38.70 & & 32.32 & 22.86 & 22.75 & - & - & 36.13 & - & - & - & 39.40 & 20.38 \\
\hline Th & - & 14.73 & & 11.03 & 15.58 & 25.66 & - & - & 41.75 & - & - & - & 42.20 & 6.24 \\
\hline $\mathrm{U}$ & - & 2.28 & & 2.25 & 2.27 & 3.09 & - & - & 2.43 & - & - & - & 2.75 & 1.37 \\
\hline $\mathrm{La}$ & - & 46.77 & & 22.73 & 26.62 & 37.78 & - & - & 123.12 & - & - & - & 127.85 & 17.01 \\
\hline $\mathrm{Ce}$ & - & 81.12 & & 49.08 & 48.01 & 72.74 & - & - & 219.89 & - & - & - & 232.58 & 31.80 \\
\hline $\operatorname{Pr}$ & - & 9.29 & & 5.58 & 6.49 & 7.96 & - & - & 20.65 & - & - & - & 28.92 & 3.87 \\
\hline $\mathrm{Nd}$ & - & 34.15 & & 20.77 & 23.37 & 28.08 & - & - & 90.76 & - & - & - & 107.44 & 13.97 \\
\hline $\mathrm{Sm}$ & - & 5.69 & & 4.13 & 4.11 & 4.50 & - & - & 15.12 & - & - & - & 19.24 & 2.42 \\
\hline $\mathrm{Eu}$ & - & 1.60 & & 0.69 & 0.50 & 0.50 & - & - & 3.81 & - & - & - & 4.69 & 0.63 \\
\hline $\mathrm{Gd}$ & - & 5.69 & & 4.29 & 3.76 & 4.14 & - & - & 14.96 & - & - & - & 18.24 & 2.42 \\
\hline $\mathrm{Tb}$ & - & 0.89 & & 0.93 & 0.71 & 0.64 & - & - & 1.46 & - & - & - & 2.83 & 0.36 \\
\hline Dy & - & 5.18 & & 6.68 & 4.50 & 3.67 & - & - & 12.73 & - & - & - & 15.47 & 2.07 \\
\hline Ho & - & 0.93 & & 1.28 & 0.82 & 0.64 & - & - & 2.2 & - & - & - & 2.67 & 0.35 \\
\hline $\mathrm{Er}$ & - & 2.87 & & 4.08 & 2.70 & 2.10 & - & - & 6.38 & - & - & - & 7.99 & 1.19 \\
\hline $\mathrm{Tm}$ & - & 0.36 & & 0.59 & 0.39 & 0.30 & - & - & 0.77 & - & - & - & 0.91 & 0.15 \\
\hline $\mathrm{Yb}$ & - & 2.66 & & 4.79 & 3.16 & 2.40 & - & - & 4.72 & - & - & - & 6.24 & 1.07 \\
\hline $\mathrm{Lu}$ & - & 0.34 & & 0.58 & 0.40 & 0.32 & - & - & 0.61 & - & - & - & 0.76 & 0.13 \\
\hline $\mathrm{Th} / \mathrm{U}$ & - & 6.46 & & 4.91 & 6.87 & 8.32 & - & - & 17.18 & - & - & - & 15.36 & 4.55 \\
\hline $\mathrm{La} / \mathrm{Th}$ & - & 3.18 & & 2.06 & 1.71 & 1.47 & - & - & 2.95 & - & - & - & 3.03 & 2.73 \\
\hline $\mathrm{La} / \mathrm{Co}$ & - & 5.66 & & 2.14 & 4.54 & 3.19 & - & - & 8.15 & - & - & - & 8.54 & 2.38 \\
\hline $\mathrm{Th} / \mathrm{Co}$ & - & 1.78 & & 1.04 & 2.65 & 2.17 & - & - & 2.76 & - & - & - & 2.82 & 0.87 \\
\hline $\mathrm{La} / \mathrm{Sc}$ & - & 6.61 & & 2.56 & 2.53 & 3.09 & - & - & 8.37 & - & - & - & 8.35 & 3.47 \\
\hline $\mathrm{Th} / \mathrm{Sc}$ & - & 2.08 & & 1.24 & 1.48 & 2.10 & - & - & 2.84 & - & - & - & 2.75 & 1.27 \\
\hline $\mathrm{Th} / \mathrm{Cr}$ & - & 0.90 & & 0.62 & 0.53 & 1.14 & - & - & 2.14 & - & - & - & 2.22 & 0.63 \\
\hline $\mathrm{Rb} / \mathrm{Sr}$ & - & 0.26 & & 0.50 & 0.63 & 0.79 & - & - & 0.25 & - & - & - & 0.25 & 0.02 \\
\hline $\mathrm{Cr} / \mathrm{Th}$ & - & 1.12 & & 1.63 & 1.88 & 0.88 & - & - & 0.47 & - & - & - & 0.45 & 1.58 \\
\hline LREE/HREE & - & 8.63 & & 4.28 & 6.41 & 10.28 & - & - & 9.98 & - & - & - & 8.63 & 8.27 \\
\hline$\Sigma$ REE & - & 197.54 & & 126.16 & 125.53 & 165.75 & - & - & 517 & - & - & - & 575.81 & 77.42 \\
\hline$(\mathrm{La} / \mathrm{Yb})_{\mathrm{cn}}$ & - & 11.89 & & 3.21 & 5.69 & 10.65 & - & - & 17.63 & - & & - & 13.84 & 10.78 \\
\hline$(\mathrm{La} / \mathrm{Sm})_{\mathrm{cn}}$ & - & 5.17 & & 3.47 & 4.08 & 5.29 & - & - & & - & & - & 4.18 & 4.43 \\
\hline$(\mathrm{Gd} / \mathrm{Yb})_{\mathrm{cn}}$ & - & 1.73 & & 0.73 & 0.96 & 1.40 & - & - & 2.57 & - & - & - & 2.37 & 1.84 \\
\hline $\mathrm{Eu} / \mathrm{Eu}^{*}$ & - & 0.86 & & 0.50 & 0.38 & 0.35 & - & - & 0.77 & - & - & - & 0.77 & 0.79 \\
\hline
\end{tabular}


Table 2 Contd....

\begin{tabular}{|c|c|c|c|c|c|c|c|c|c|c|c|c|c|c|c|c|}
\hline \multirow{2}{*}{$\begin{array}{l}\text { Rock type } \\
\text { Sample \# }\end{array}$} & \multicolumn{4}{|c|}{ Litharenites } & \multirow{2}{*}{$\begin{array}{c}\text { Sublith } \\
\text { arenite } \\
\text { SB }\end{array}$} & \multicolumn{7}{|c|}{ Subarkoses } & \multicolumn{2}{|c|}{ Arkose } & \multicolumn{2}{|c|}{ Wacke } \\
\hline & VP-I & $\mathrm{KN}$ & $\mathrm{KR}$ & $\mathrm{TM}$ & & SK-I & Sk-VII & SK-IX & SK-X & SK-XII & $\mathrm{KD}$ & KK & MM & MM-I & $\mathrm{KL}$ & KY \\
\hline $\mathrm{SiO}_{2}$ & 63.49 & 69.37 & 67.94 & 63.19 & 68.45 & 77.18 & 70.38 & 79.21 & 70.24 & 68.97 & 65.79 & 68.61 & 62.74 & 61.82 & 60.59 & 61.95 \\
\hline $\mathrm{TiO}_{2}$ & 1.06 & 0.98 & 0.97 & 0.97 & 0.98 & 1.38 & 1.36 & 1.31 & 1.06 & 1.17 & 0.72 & 0.99 & 0.91 & 0.91 & 0.97 & 0.96 \\
\hline $\mathrm{Al}_{2} \mathrm{O}_{3}$ & 5.20 & 8.91 & 9.29 & 6.59 & 4.65 & 4.34 & 4.67 & 1.86 & 2.27 & 3.67 & 1.46 & 3.40 & 9.89 & 9.62 & 9.94 & 10.70 \\
\hline $\mathrm{Fe}_{2} \mathrm{O}_{3}$ & 5.91 & 1.72 & 1.36 & 2.15 & 1.82 & 0.37 & 0.08 & 0.61 & 0.12 & 1.04 & 2.90 & 1.70 & 1.68 & 1.52 & 2.01 & 1.53 \\
\hline $\mathrm{MnO}$ & 0.012 & 0.027 & 0.077 & 0.181 & 0.097 & 0.008 & 0.033 & 0.015 & 0.612 & 0.814 & 0.015 & 0.133 & 0.099 & 0.088 & 0.209 & 0.081 \\
\hline $\mathrm{MgO}$ & 0.16 & 0.29 & 0.28 & 0.25 & 0.24 & 0.26 & 0.27 & 0.20 & 0.26 & 0.27 & 0.19 & 0.29 & 0.21 & 0.21 & 0.23 & 0.36 \\
\hline $\mathrm{CaO}$ & 14.70 & 10.21 & 11.90 & 16.61 & 10.55 & 8.30 & 13.70 & 7.60 & 13.90 & 13.10 & 15.32 & 11.86 & 12.64 & 12.43 & 13.93 & 12.32 \\
\hline $\mathrm{Na}_{2} \mathrm{O}$ & 0.61 & 1.52 & 2.11 & 1.77 & 1.21 & 0.31 & 1.63 & 0.37 & 0.63 & 1.29 & 1.53 & 3.20 & 2.74 & 2.72 & 1.28 & 1.04 \\
\hline $\mathrm{K}_{2} \mathrm{O}$ & 1.53 & 0.72 & 0.95 & 0.77 & 1.39 & 2.66 & 2.35 & 2.67 & 3.26 & 3.83 & 3.66 & 4.21 & 3.03 & 3.45 & 1.02 & 0.74 \\
\hline $\mathrm{P}_{2} \mathrm{O}_{5}$ & 0.31 & 0.25 & 0.39 & 0.26 & 0.26 & 0.15 & 0.29 & 0.16 & 0.29 & 0.31 & 0.13 & 0.35 & 0.24 & 0.29 & 0.17 & 0.31 \\
\hline LOI & 8.21 & 7.13 & 6.02 & 8.21 & 8.97 & 5.13 & 6.14 & 5.10 & 6.98 & 6.01 & 8.27 & 6.20 & 6.21 & 6.07 & 8.76 & 10.08 \\
\hline Total & 101.19 & 101.13 & 101.28 & 100.95 & 98.62 & 100.09 & 100.90 & 99.11 & 99.62 & 100.47 & 99.97 & 100.95 & 100.38 & 99.13 & 99.10 & 100.07 \\
\hline $\mathrm{Sc}$ & 7.54 & 20.75 & 19.19 & 12.46 & 18.80 & 8.95 & 8.50 & - & - & 9.12 & 9.76 & - & 11.29 & 11.09 & 12.03 & 18.03 \\
\hline $\mathrm{V}$ & 74.49 & 90.81 & 121.71 & 66.76 & 125.84 & 105.14 & 90.60 & - & - & 91.33 & 105.13 & - & 82.48 & 82.54 & 80.73 & 87.84 \\
\hline $\mathrm{Cr}$ & 28.43 & 26.06 & 28.79 & 24.03 & 33.01 & 19.71 & 18.96 & - & - & 18.51 & 20.34 & - & 20.81 & 20.67 & 17.99 & 18.32 \\
\hline Co & 9.25 & 7.38 & 8.92 & 6.30 & 29.43 & 12.35 & 11.14 & - & - & 10.82 & 37.46 & - & 9.35 & 9.72 & 6.81 & 17.67 \\
\hline $\mathrm{Ni}$ & 29.08 & 21.23 & 25.26 & 23.39 & 43.80 & 32.46 & 24.97 & - & - & 24.32 & 51.43 & - & 20.75 & 19.42 & 21.18 & 30.06 \\
\hline $\mathrm{Cu}$ & 26.63 & 21.90 & 20.68 & 15.34 & 25.66 & 26.16 & 25.49 & - & - & 25.23 & 18.40 & - & 16.03 & 16.38 & 16.47 & 21.56 \\
\hline $\mathrm{Zn}$ & 35.50 & 50.42 & 73.62 & 31.88 & 57.84 & 48.03 & 46.19 & - & - & 45.47 & 30.55 & - & 38.20 & 37.9 & 35.20 & 45.55 \\
\hline $\mathrm{Ga}$ & 17.32 & 19.84 & 24.30 & 16.65 & 17.40 & 18.83 & 19.70 & - & - & 18.90 & 10.32 & - & 23.80 & 22.76 & 14.38 & 18.58 \\
\hline $\mathrm{Rb}$ & 55.75 & 44.11 & 71.90 & 99.43 & 38.86 & 80.44 & 83.85 & - & - & 80.32 & 23.12 & - & 148.42 & 148.1 & 80.56 & 91.55 \\
\hline $\mathrm{Sr}$ & 291.31 & 501.87 & 530.81 & 406.39 & 447.25 & 234.13 & 229.43 & - & - & 222.43 & 254.67 & - & 451.15 & 450.8 & 281.56 & 232.95 \\
\hline $\mathrm{Y}$ & 37.30 & 20.30 & 37.94 & 33.40 & 68.23 & 41.27 & 41.27 & - & - & 40.53 & 90.44 & - & 15.57 & 16.02 & 37.07 & 40.13 \\
\hline $\mathrm{Zr}$ & 2775.36 & 292.74 & 506.35 & 248.29 & 319.92 & 2676.02 & 5892.03 & - & - & 5709.84 & 172.09 & - & 204.17 & 202 & 386.19 & 324.98 \\
\hline $\mathrm{Nb}$ & 9.08 & 11.30 & 28.01 & 11.91 & 11.11 & 14.79 & 24.43 & - & - & 22.69 & 4.17 & - & 7.96 & 6.47 & 14.75 & 16.73 \\
\hline Cs & 1.87 & 0.38 & 1.20 & 0.97 & 0.50 & 1.93 & 2.11 & - & - & 2.03 & 0.56 & - & 1.55 & 1.09 & 0.74 & 1.25 \\
\hline $\mathrm{Ba}$ & 3843.43 & 1577.22 & 1501.02 & 2962.48 & 1553.25 & 4170.94 & 950.27 & - & - & 931.15 & 1720.16 & - & 1822.48 & 1797 & 1772.33 & 1455.00 \\
\hline $\mathrm{Hf}$ & 80.75 & 8.96 & 15.50 & 0.77 & 10.19 & 75.59 & 83.64 & - & - & 80.46 & 5.31 & - & 5.54 & 5.21 & 11.06 & 9.38 \\
\hline $\mathrm{Ta}$ & 0.33 & 1.28 & 1.88 & 0.85 & 0.65 & 1.06 & 1.50 & - & - & 1.50 & 0.10 & - & 0.46 & 0.54 & 1.10 & 1.37 \\
\hline $\mathrm{Pb}$ & 33.92 & 25.46 & 32.39 & 21.01 & 28.02 & 34.59 & 24.45 & - & - & 27.16 & 24.12 & - & 45.65 & 30.87 & 19.74 & 24.41 \\
\hline Th & 8.35 & 36.15 & 38.53 & 14.25 & 7.71 & 30.63 & 55.81 & - & - & 50.32 & 5.29 & - & 7.24 & 7.65 & 23.85 & 19.20 \\
\hline $\mathrm{U}$ & 2.16 & 1.83 & 2.49 & 1.04 & 0.88 & 3.04 & 3.36 & - & - & 3.56 & 1.16 & - & 1.15 & 1.26 & 1.82 & 2.32 \\
\hline $\mathrm{La}$ & 31.23 & 58.69 & 82.92 & 39.28 & 36.34 & 76.21 & 79.59 & - & - & 77.71 & 58.83 & - & 36.17 & 33.23 & 53.90 & 45.30 \\
\hline $\mathrm{Ce}$ & 55.38 & 104.45 & 160.39 & 75.00 & 58.90 & 142.26 & 155.09 & - & - & 151.60 & 80.74 & - & 65.02 & 60.38 & 103.43 & 89.58 \\
\hline $\operatorname{Pr}$ & 6.71 & 11.44 & 19.85 & 8.58 & 8.22 & 17.66 & 18.04 & - & - & 17.47 & 10.81 & - & 7.10 & 6.14 & 12.16 & 9.95 \\
\hline $\mathrm{Nd}$ & 25.86 & 39.35 & 71.55 & 30.71 & 32.63 & 64.51 & 65.50 & - & - & 63.55 & 42.72 & - & 24.11 & 23.86 & 43.67 & 35.59 \\
\hline $\mathrm{Sm}$ & 4.71 & 6.35 & 12.07 & 5.56 & 6.75 & 10.77 & 10.45 & - & - & 10.17 & 8.29 & - & 4.03 & 4.12 & 7.52 & 6.14 \\
\hline $\mathrm{Eu}$ & 1.67 & 1.69 & 2.49 & 2.18 & 2.38 & 2.63 & 1.44 & - & - & 1.42 & 2.74 & - & 2.21 & 1.67 & 1.68 & 1.65 \\
\hline $\mathrm{Gd}$ & 5.13 & 5.91 & 10.64 & 5.72 & 7.92 & 10.03 & 9.51 & - & - & 9.04 & 9.47 & - & 3.67 & 4.12 & 7.07 & 6.45 \\
\hline $\mathrm{Tb}$ & 0.88 & 0.81 & 1.47 & 0.90 & 1.47 & 1.46 & 1.34 & - & - & 1.32 & 1.65 & - & 0.55 & 0.88 & 1.10 & 1.06 \\
\hline Dy & 5.56 & 4.05 & 7.80 & 5.57 & 9.88 & 7.96 & 7.30 & - & - & 7.23 & 11.05 & - & 3.16 & 3.56 & 6.46 & 5.68 \\
\hline Ho & 1.04 & 0.66 & 1.27 & 0.97 & 1.84 & 1.38 & 1.25 & - & - & 1.19 & 2.08 & - & 0.52 & 0.65 & 1.11 & 1.22 \\
\hline $\mathrm{Er}$ & 3.13 & 2.18 & 4.09 & 3.08 & 5.70 & 4.23 & 3.83 & - & - & 3.91 & 6.38 & - & 1.62 & 1.13 & 3.53 & 4.05 \\
\hline $\mathrm{Tm}$ & 0.40 & 0.27 & 0.50 & 0.39 & 0.72 & 0.53 & 0.48 & - & - & 0.45 & 0.75 & - & 0.21 & 0.29 & 0.45 & 0.55 \\
\hline $\mathrm{Yb}$ & 2.89 & 1.99 & 3.89 & 2.81 & 5.00 & 3.98 & 3.67 & - & - & 3.45 & 5.24 & - & 1.67 & 1.89 & 3.41 & 4.15 \\
\hline $\mathrm{Lu}$ & 0.38 & 0.26 & 0.47 & 0.35 & 0.65 & 0.49 & 0.46 & - & - & 0.44 & 0.66 & - & 0.20 & 0.28 & 0.42 & 0.54 \\
\hline $\mathrm{Th} / \mathrm{U}$ & 3.86 & 19.73 & 15.48 & 13.77 & 8.75 & 10.06 & 16.60 & & - & 14.14 & 4.57 & - & 6.28 & 6.07 & 13.08 & 8.27 \\
\hline $\mathrm{La} / \mathrm{Th}$ & 3.74 & 1.62 & 2.15 & 2.76 & 4.72 & 2.49 & 1.43 & & - & 1.54 & 11.12 & - & 4.99 & 4.34 & 2.26 & 2.36 \\
\hline $\mathrm{La} / \mathrm{Co}$ & 3.38 & 7.95 & 9.29 & 6.23 & 1.23 & 6.17 & 7.15 & & - & 7.18 & 1.57 & - & 3.87 & 3.42 & 7.91 & 2.56 \\
\hline Th/Co & 0.90 & 4.90 & 4.32 & 2.26 & 0.26 & 2.48 & 5.01 & & - & 4.65 & 0.14 & - & 0.77 & 0.79 & 3.50 & 1.09 \\
\hline $\mathrm{La} / \mathrm{Sc}$ & 4.14 & 2.83 & 4.32 & 3.15 & 1.93 & 8.51 & 9.36 & & - & 8.52 & 6.03 & - & 3.20 & 3.00 & 4.48 & 2.51 \\
\hline $\mathrm{Th} / \mathrm{Sc}$ & 1.11 & 1.74 & 2.01 & 1.14 & 0.41 & 3.42 & 6.57 & & - & 5.52 & 0.54 & - & 0.64 & 0.69 & 1.98 & 1.06 \\
\hline $\mathrm{Th} / \mathrm{Cr}$ & 0.29 & 1.39 & 1.34 & 0.59 & 0.23 & 1.55 & 2.94 & & - & 2.72 & 0.26 & - & 0.35 & 0.37 & 1.33 & 1.05 \\
\hline $\mathrm{Rb} / \mathrm{Sr}$ & 0.19 & 0.09 & 0.14 & 0.24 & 0.09 & 0.34 & 0.37 & & - & 0.36 & 0.09 & - & 0.33 & 0.33 & 0.29 & 0.39 \\
\hline $\mathrm{Cr} / \mathrm{Th}$ & 3.41 & 0.72 & 0.75 & 1.69 & 4.28 & 0.64 & 0.34 & & - & 0.37 & 3.84 & - & 2.87 & 2.07 & 0.75 & 0.95 \\
\hline LREE/HREE & 5.88 & 12.36 & 10.63 & 7.24 & 4.02 & 9.53 & 11.23 & - & - & 11.27 & 5.04 & - & 9.87 & 8.83 & 8.75 & 7.36 \\
\hline$\Sigma \mathrm{REE}$ & 144.97 & 238.09 & 379.40 & 181.10 & 178.38 & 344.10 & 357.94 & - & - & 348.96 & 241.38 & - & 150.25 & 142.20 & 245.90 & 211.89 \\
\hline$(\mathrm{La} / \mathrm{Yb})_{\mathrm{cn}}$ & 7.29 & 19.90 & 14.40 & 9.45 & 4.91 & 12.94 & 14.65 & & & 15.23 & 7.59 & & 14.65 & 14.56 & 10.68 & 7.38 \\
\hline$(\mathrm{La} / \mathrm{Sm})_{\mathrm{cn}}$ & 4.17 & 5.82 & 4.33 & 4.45 & 3.39 & 4.46 & 4.79 & & & 4.81 & 4.47 & & 5.65 & 5.58 & 4.51 & 4.65 \\
\hline$(\mathrm{Gd} / \mathrm{Yb})_{\mathrm{cn}}$ & 1.44 & 2.40 & 2.22 & 1.65 & 1.28 & 2.04 & 2.10 & & - & 2.12 & 1.46 & - & 1.79 & 1.62 & 1.68 & 1.26 \\
\hline $\mathrm{Eu} / \mathrm{Eu}^{*}$ & 1.04 & 0.84 & 0.67 & 1.18 & 0.99 & 0.77 & 0.44 & & - & 0.45 & 0.94 & - & 1.76 & 1.67 & 0.70 & 0.80 \\
\hline
\end{tabular}




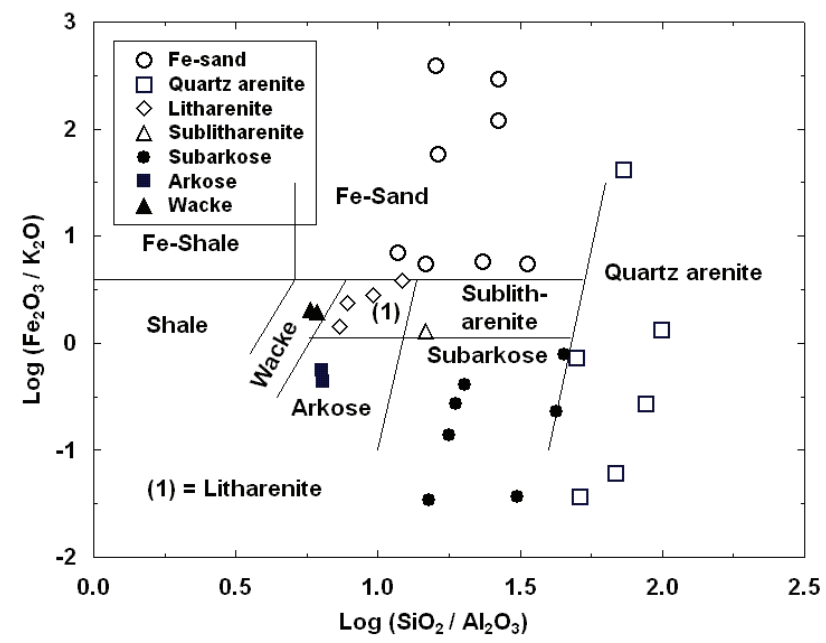

Fig.2. Geochemical classification of Sillakkudi sandstones using $\log \left(\mathrm{SiO}_{2} / \mathrm{Al}_{2} \mathrm{O}_{3}\right)-\log \left(\mathrm{Fe}_{2} \mathrm{O}_{3} * / \mathrm{K}_{2} \mathrm{O}\right)$ diagram (after Herron, 1988).

contents and their ratios $\left(\mathrm{K}_{2} \mathrm{O} / \mathrm{Na}_{2} \mathrm{O} \geq 1\right.$ or $\left.<1\right)$ are also consistent with the petrographic observations, according to which K-feldspar dominates over plagioclase feldspar and $\mathrm{K}_{2} \mathrm{O} / \mathrm{Na}_{2} \mathrm{O}<1$ in some samples indicate predominance of plagioclase over K-feldspar.

Concentrations of trace elements in Sillakkudi sandstones (Table 2) are generally lower than the average upper continental crust (UCC) concentrations and near to 1 (Fig. 3). The Large ion lithophile elements (LILE) like Rb, $\mathrm{Ba}$, and $\mathrm{Sr}$ are compared with UCC in which $\mathrm{Ba}$ is higher in all sandstone types. However, $\mathrm{Sr}$ is higher in sub-litharenite, quartz arenite, and wacke types than sub-arkose and fe-sands. The reverse trend is observed in Rb concentration, which is higher in wacke than the other sandstone types. The transition trace elements $\mathrm{Co}, \mathrm{Ni}, \mathrm{V}$ are higher and $\mathrm{Cr}$ is lower in the Sillakkudi sandstones than UCC. The significant enrichment of $\mathrm{Zr}$, Hf and Th in sub-arkose, sub-litharenite, fe-sand and

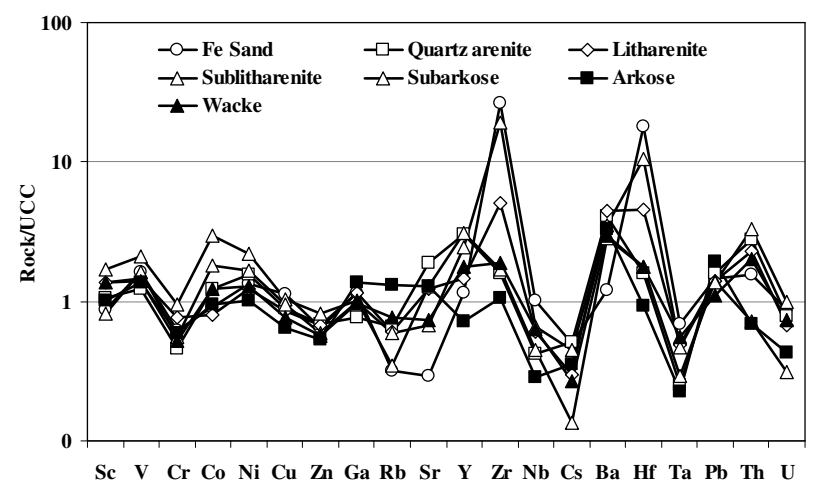

Fig.3. Multi-element normalized diagram for the Sillakkudi sandstones, normalized against average upper continental crust values (UCC; Taylor and McLennan, 1985).

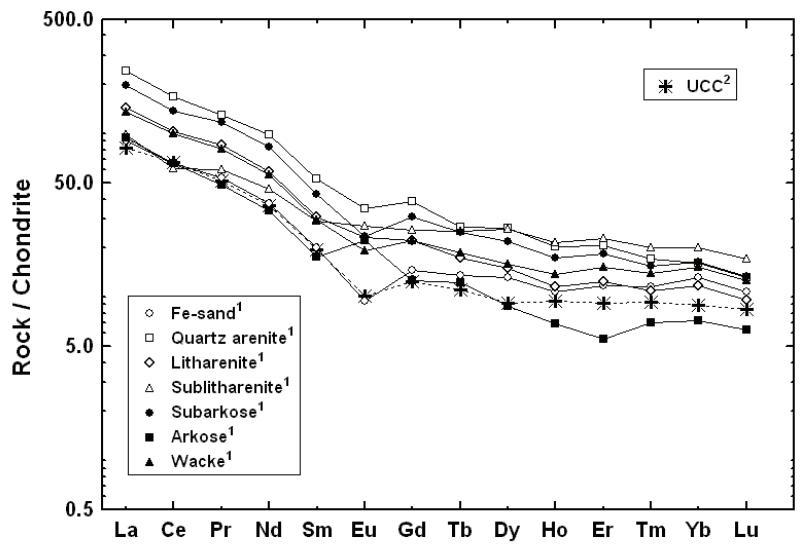

Fig.4. Chondrite normalized rare earth elements plot for the Sillakkudi sandstones. Chondrite normalization and upper continental crust values are from Taylor and McLennan (1985) ${ }^{1}$ This study, ${ }^{2}$ Taylor and McLennan (1985).

quartz arenite could be related to the amount of heavy minerals (especially zircon) present in them. The high abundance of $\mathrm{Zr}$ (93-5892 ppm) in the Sillakkudi sandstones indicate that they may be derived from zircon enriched source rocks such as massif charnockites and granitoids from Dharwar Craton.

The REE concentrations of the Sillakkudi sandstones are also given in the Table 2 and are shown as chondrite normalized patterns in Fig.4. Total REE concentrations vary widely in Sillakkudi sandstones (77-576; mean $=247$ ppm). The wide variations are also identified in individual rock types, e.g., fe-sand ( 126-197 ppm; $\mathrm{n}=4)$ quartz arenite $(77-573 ; \mathrm{n}=3)$ and litharenite $(\sim 145-379 ; \mathrm{n}=4)$. The average $\Sigma$ REE concentrations are higher in the Sillakkudi sandstones than the UCC value ( 143; Taylor and McLennan 1985). The lower REE content in quartz arenite (77 ppm; sample no. KP; Table 2) than other sandstone types highlight a possible paucity of clays and/or quartz dilution effect caused by sedimentary sorting. The REE patterns (Fig. 4) of the Sillakkudi sandstones are with enriched $\operatorname{LREE}\left(\mathrm{La}_{\mathrm{CN}} / \mathrm{Sm}_{\mathrm{CN}}=4.6 \pm 0.6 ; \mathrm{n}=20\right)$, relatively flat HREE $\left(\mathrm{Gd}_{\mathrm{CN}} / \mathrm{Yb}_{\mathrm{CN}}=1.74 \pm 0.50\right)$ and a negative Eu anomaly $\left(\mathrm{Eu} / \mathrm{Eu}^{*}=0.80 \pm 0.33\right)$.

\section{DISCUSSION}

Based on the petrographic study the Sillakkudi sandstones are classified as quartz arenite, litharenite, arkose, sub-arkose, wacke and iron rich sandstone (ferrugenous sandstone) types. The average contents of different quartz grains in these sandstones show granitic and/or gneissic source. This has been confirmed by overall variation in the relative abundance of different types of quartz grains 
[monocrystalline (undulatory and non undulatory) and polycrystalline]. The greater abundance of alkali feldspar than plagioclase further supports for granitic and/or gneissic source. Glauconites are common in some sandstone types, which indicate shallow marine environments. Several authors (Pettijohn et al. 1972; Blatt et al. 1980; Herron, 1988) have proposed the geochemical classification diagrams for terrigenous sedimentary rocks using major elements. However, the modified classification diagram proposed by Herron (1988) facilitates arkoses to be more successfully classified than Pettijohn et al. (1972) diagram and it is also a measure of mineral stability as the ferromagnesian minerals tend to be amongst the least stable minerals during weathering. Using the geochemical classification diagram of Herron (1988) the Sillakkudi sandstones are classified as fe-sand, quartz arenite, litharenite, sub-litharenite, sub-arkose, arkose, and wacke types (Fig.2). This classification is supported petrographically in which fe-sands are classified as ferruginous sandstone.

\section{Tectonic Setting}

The functioning of different tectonic setting discrimination diagrams proposed by Bhatia (1983) and Roser and Korsch (1986) using major elements have been evaluated by many studies (e.g., Armstrong-Altrin and Verma, 2005; Ryan and Williams, 2007). Armstrong-Altrin and Verma (2005) observed that the tectonic setting discrimination diagram proposed by Roser and Korsch (1986) works better than Bhatia's (1983) diagrams to determine the plate tectonic setting of sedimentary basins. $\mathrm{K}_{2} \mathrm{O} / \mathrm{Na}_{2} \mathrm{O}-\mathrm{SiO}_{2}$ diagram (Fig.5) is used here after recalculating the analyses $100 \%$ volatile-free (Roser and Korsch, 1986). This has been done in case of those samples, which contain a considerable amount of $\mathrm{CaO}(<20 \%)$, much of it representing $\mathrm{CaCO}_{3}$ in order to compare the data closely. Data in this case could not be calculated carbonate-free since $\mathrm{CO}_{2}$ values are not available; hence it was recalculated to $100 \% \mathrm{CaO}$ free. On this diagram, (Fig.5), the Sillakkudi sandstones plot in the passive and active continental margin fields. The shifting of samples towards the ACM field is due to their low $\mathrm{K}_{2} \mathrm{O} / \mathrm{Na}_{2} \mathrm{O}$ ratio and high $\mathrm{SiO}_{2}$ content (Table 2). Similarly, REE distribution in sandstones are also used to infer the tectonic settings by various authors (e.g. McLennan et al. 1990; McLennan and Taylor, 1991; Asiedu et al. 2000). According to these studies, the sediments deposited in the continental margin are characterized by LREE enrichment (high $\mathrm{La}_{\mathrm{CN}} / \mathrm{Sm}_{\mathrm{CN}}$ ) and high total rare earth elements ( $\mathrm{REE}$ ), whereas sediments deposited from young, undifferentiated oceanic arcs have low $\left(\mathrm{La}_{\mathrm{CN}} / \mathrm{Sm}_{\mathrm{CN}}\right)$

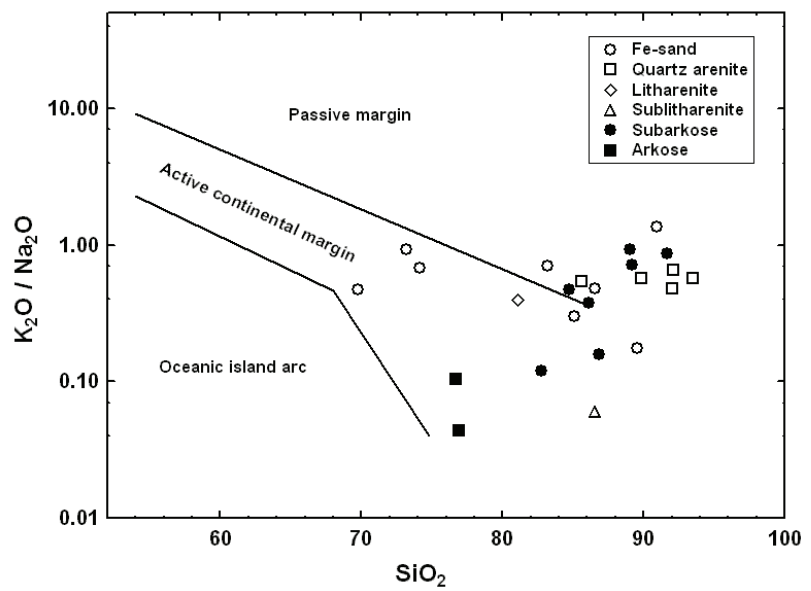

Fig.5. $\mathrm{K}_{2} \mathrm{O} / \mathrm{Na}_{2} \mathrm{O}$ versus $\mathrm{SiO}_{2}$ tectonic setting discrimination diagram for the Sillakkudi sandstones (after Roser and Korsch, 1986; the samples with very low $\mathrm{K}_{2} \mathrm{O} / \mathrm{Na}_{2} \mathrm{O}$ ratio are not included).

ratio, $\Sigma$ REE content, and lack of negative Eu anomaly. Passive margin provenance is typically characterized by uniform REE pattern similar to PAAS (Bhatia, 1985; McLennan, 1989), while sediments from active continental margin display intermediate $\Sigma$ REE abundances with variable negative Eu anomaly in the range of 0.6-1.0 (McLennan, 1989). In this study, sandstones have relatively high $\mathrm{La}_{\mathrm{CN}} / \mathrm{Sm}_{\mathrm{CN}}(5.39-9.25 ; 7.33 \pm 1), \mathrm{La}_{\mathrm{CN}} / \mathrm{Yb}_{\mathrm{CN}}$ (3.21-19.90; $11.23 \pm 4.34)$ and variable $\mathrm{Eu} / \mathrm{Eu}^{*}$ ratios $(0.35-1.73 ; 0.80 \pm$ 0.33 ) suggesting a combination of passive and active margin settings.

\section{Weathering and Hydraulic Sorting}

Alteration of sedimentary rocks can be determined by various parameters like chemical index of alteration (CIA, Nesbitt and Young, 1982), $\mathrm{K}_{2} \mathrm{O} / \mathrm{Na}_{2} \mathrm{O}$ (Nesbitt and Young, 1984; Lindsey, 1999; Dey et al. 2009), Rb/Sr (McLennan et al. 1993), and Th/U ratios (McLennan et al. 1993). These parameters have been successfully used in several studies (e.g. Hurowitz and McLennan, 2005; Selvaraj and Chen, 2006; van Staden et al. 2006; Varga et al. 2007; Pe-Piper et al. 2008; Zimmermann and Spalletti, 2009; Wani and Mondal, 2010) to identify the intensity of weathering.

The average CIA values are higher in fe-sands (78), wacke (76), and litharenite (65) than sub-litharenite (55), arkose (53), sub-arkose (36), and quartz arenite (31). Unweathered igneous rocks have CIA values close to 50, whereas intensely weathered residual rock forming the minerals kaolinite and gibbsite has values 100 (Nesbitt and Young, 1982). However, the average CIA values are consistent with UCC value, which indicate moderate weathering in the source region. The low average CIA values 
in quartz arenite, arkose and sub-arkose are due to direct input of immature continent detrital minerals in to the depositional system and do not reflect the general chemical weathering conditions in the source region, which can be inferred from other geochemical indices. Similarly, the variations in CIA values also indicate the sediment sorting effect. Physical sorting of sediments during transportation and deposition leads to concentration of quartz and feldspar with some heavy minerals in the coarse fraction and more weatherable minerals in the suspended load sediments (Garcia et al. 2004).

The average $\mathrm{K}_{2} \mathrm{O} / \mathrm{Na}_{2} \mathrm{O}$ ratios in fe-sand $(6 \pm 7)$, quartz arenite $(3.16 \pm 1.39)$, litharenite $(0.97 \pm 1.03)$, sub-litharenite (1.14), sub-arkose $(4.16 \pm 2.90)$, arkose $(1.89 \pm 0.19)$ and wacke $(0.75 \pm 0.06)$ indicates the destruction of plagioclase is higher than the K-feldspar. Th/ $\mathrm{U}$ ratio is also useful to study the source characteristics of clastic sedimentary rocks (e.g. Rodazz et al. 2006). The higher $\mathrm{Th} / \mathrm{U}$ ratios in the sediments are due to oxidative weathering, and removal of $\mathrm{U}$ and $\mathrm{Th} / \mathrm{U}$ increases with kaolinite content in highly weathered rocks (McLennan et al. 1980). Clastic sedimentary rocks derived from the upper crust are characterized by $\mathrm{Th} / \mathrm{U}$ ratios equal to or greater than 4 , whereas $\mathrm{Th} / \mathrm{U}$ ratios lower than 4 have been related to mantle contribution (Roddaz et al. 2006). Figure 6 shows that the $\mathrm{Th} / \mathrm{U}$ ratios in the studied samples are higher than 4 and indicates moderate intensity of weathering. $\mathrm{Rb} / \mathrm{Sr}$ ratio of sediments can also monitor the degree of source rock weathering (McLennan et al. 1993). Because, chemical weathering of the source area produces higher $\mathrm{Rb} / \mathrm{Sr}$ ratios in sedimentary rocks (Kimberley and Grandstaff, 1986). The average $\mathrm{Rb} / \mathrm{Sr}$ ratio of Sillakkudi sandstones is 0.30 , which is close to the average UCC value (0.32; Taylor and McLennan, 1985) but significantly lower than the PAAS value (0.80; McLennan et al. 1983). This suggests that the intensity of source area weathering was most probably moderate rather than intense.

Hydraulic sorting can significantly influence the chemical composition of terrigenous sediments, and have control on the distribution of some major elements $\left(\mathrm{P}_{2} \mathrm{O}_{5}\right.$ and $\mathrm{TiO}_{2}$ ) and trace elements (e.g., REE, Th, U, Zr, Hf and $\mathrm{Nb})$. The average $\mathrm{Zr}$ content (1549 ppm) in Sillakkudi sandstones is higher than PAAS (210; McLennan et al., 1983) and UCC (190; Taylor and McLennan, 1985) values. $\mathrm{Zr}$ and $\mathrm{Hf}$ behave similar as attested by their positive correlation coefficient $(\mathrm{r}=0.69, \mathrm{n}=20$ ). The $\mathrm{Zr} / \mathrm{Hf}$ ratio in the Sillakkudi sandstones vary from 7 to 322 and its average $(51 \pm 65)$ is slightly higher than UCC value $(\sim 36)$. $\mathrm{Zr}$ content does not however show any distinct correlation with HREE $(r=-0.03, n=20)$, indicating that, not all the

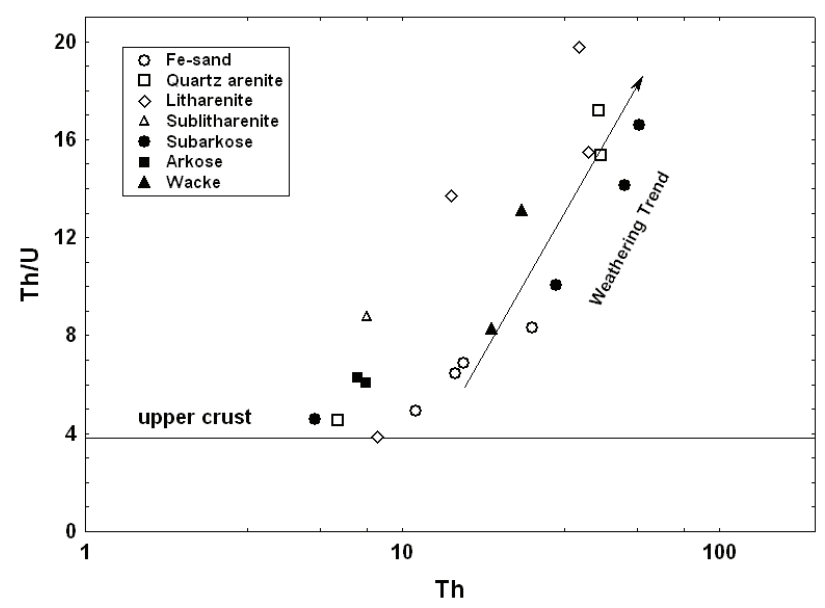

Fig.6. Th/U versus $\mathrm{Th}$ plot for the Sillakkudi sandstones constructed to identify the intensity of weathering (after McLennan et al. 1993).

HREEs are controlled by zircon abundance, although, zircon accumulation may cause enrichment in Th and HREEs. However, the poor correlations obtained for $\mathrm{Zr}$ versus $\mathrm{Th}$ $(\mathrm{r}=0.44)$ and $\mathrm{Zr}$ versus $\Sigma \mathrm{REE}(\mathrm{r}=0.08)$ indicate that zircon has little influence over the abundance of these elements. $\mathrm{Zr}$ enrichment in sediments is observed in the catchment areas of Cauvery River (Sharma and Rajamani, 2001) and identified that the increase in $\mathrm{Zr}$ content was due to the inherent heterogeneity (in granitic rocks the distribution of heavy minerals and associated trace elements are not homogeneous). If the variation of $\mathrm{Zr}$ concentration is not due to inherent heterogeneities with in rock, the factors other than weathering are considered for $\mathrm{Zr}$ enrichment (Sharma and Rajamani, 2001). The Th/Sc vs Zr/Sc plot (Fig. 7) is widely used to distinguish between the contrasting effects

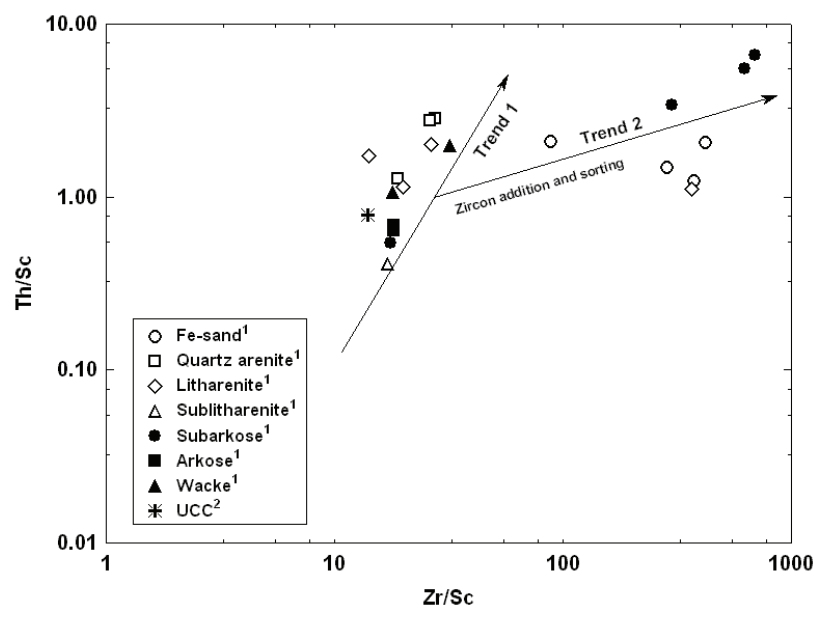

Fig.7. $\mathrm{Th} / \mathrm{Sc}$ versus $\mathrm{Zr} / \mathrm{Sc}$ diagram for the Sillakkudi sandstones (after McLennan et al. 1993). The addition of zircon due to sediment sorting and recycling is observed in Trend 2. ${ }^{1}$ This study; ${ }^{2}$ Taylor and McLennan (1985). 
of source composition and sedimentary processes on the composition of clastic sedimentary rocks (McLennan et al. 1993). $\mathrm{Zr} / \mathrm{Sc}$ ratios would increase by an addition of zircon mineral during sorting and/or recycling processes (McLennan et al., 1993). In the Th/Sc vs Zr/Sc plot (Fig. 7), trend 1 reveals the direct contribution from primary source rocks and trend 2 shows the influence of sedimentary processes. Hence, the samples which are sub-parallel to trend 1 suggests compositional homogeneity and others, which are parallel to trend 2 are indicative of heavy mineral addition during sediment recycling and sorting. Mineral sorting normally tends to increase the abundance of non-clay detrital minerals at the expense of clay minerals (Nesbitt and Young, 1984). Therefore, the relative differences in the abundance of some trace elements might have been attributed to mineral sorting (LaMaskin et al. 2008; Kasper-Zubillaga et al. 2008) and reveals that $\mathrm{Zr}$ does not control the distribution of REEs in Sillakkudi sandstones. Similarly, the negative correlation observed between $\Sigma$ REE and $\mathrm{P}_{2} \mathrm{O}_{5}$ indicates that apatite or monazite do not control the REE distribution in Sillakkudi sandstones (Condie, 1991; Das et al. 2006).

\section{Provenance}

Geochemistry of sedimentary rocks is considerable consequence to understand the provenance of the sediments (McLennan, 1989). The high field strength elements (HFSE) such as $\mathrm{Zr}$, Nb, Hf, Y, Th and $\mathrm{U}$ are preferentially partitioned into melts during crystallization (Feng and Kerrich, 1990) and as a result these elements are enriched in felsic sources rather than the mafic sources. Zr, Hf, and Y are thought to reflect provenance compositions as a consequence of their immobile behaviors (Taylor and McLennan, 1985).The ferromagnesian trace elements $\mathrm{Cr}, \mathrm{Ni}, \mathrm{Co}$ and $\mathrm{V}$ generally show similarity with the behavior of magmatic processes, but they may be fractionated during weathering (Feng and Kerrich, 1990). In the Sillakkudi sandstones, Ni is enriched whereas $\mathrm{Cr}$ is decreased with respect to the average UCC composition (Fig. 3). This enrichment in Ni may suggest some input of mafic minerals from the source terrain; however, the depletion of $\mathrm{Cr}(21 \pm 5, \mathrm{n}=30), \operatorname{MgO}(0.2 \pm$ $0.1)$ contents and $\mathrm{Cr} / \mathrm{Th}(1.57 \pm 1.22 \mathrm{n}=20)$ ratio suggests that other factors could have played a role in concentrating $\mathrm{Ni}$ in the sandstones. Similarly, no significant correlation is observed for $\mathrm{Cr}$ versus $\mathrm{Ni}(\mathrm{r}=0.08)$ and $\mathrm{Co}(\mathrm{r}=0.15)$, whereas Ni shows good positive correlation coefficient with $\mathrm{Co}(\mathrm{r}=0.92)$. Hence, the enrichment of Ni unaccompanied by $\mathrm{Cr}$ in Sillakkudi sandstones was due to the fractionation of garnet between the source rocks and sandstones, and not due to the mafic nature (Armstrong-Altrin et al. 2004).

The variations in $\mathrm{Th}$ and $\mathrm{La}$ (indicative of felsic) and,

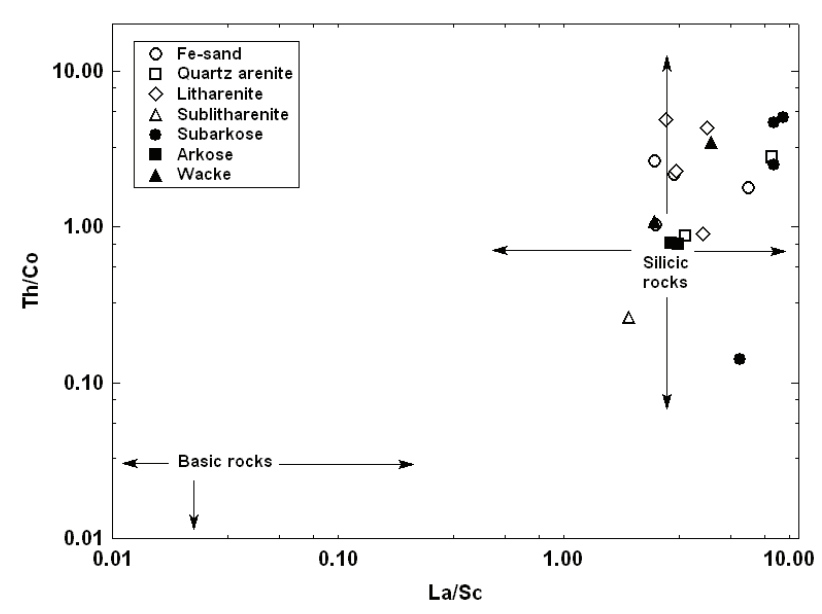

Fig.8. Th/Co versus La/Sc plot showing source rock composition for the Sillakkudi sandstones (fields after Cullers, 2002).

Sc and Co (indicative of mafic) contents have been used to differentiate between felsic and mafic provenance by various authors (McLennan et al. 1980, Cullers, 2002; Nagarajan et al. 2007a, b; Kasanzu et al. 2008). Th/Co vs La/Sc bivariate and La-Th-Sc triangular diagrams can provide information regarding the source rock characteristics (McLennan and Taylor, 1991; Cullers, 2002). The Th/Co vs La/Sc plot (Fig. 8) suggests a felsic nature of the source rocks (Cullers, 2002). Similarly, La-Th-Sc triangular diagram (Fig. 9) is also used to understand the provenance characteristics. The average compositions of granite, andesite, and basalt (Condie, 1993), and UCC are used in this diagram for comparison. In this triangular diagram (Fig. 9), sub-arkose plots near to granite composition, whereas fe-sand, arkose,

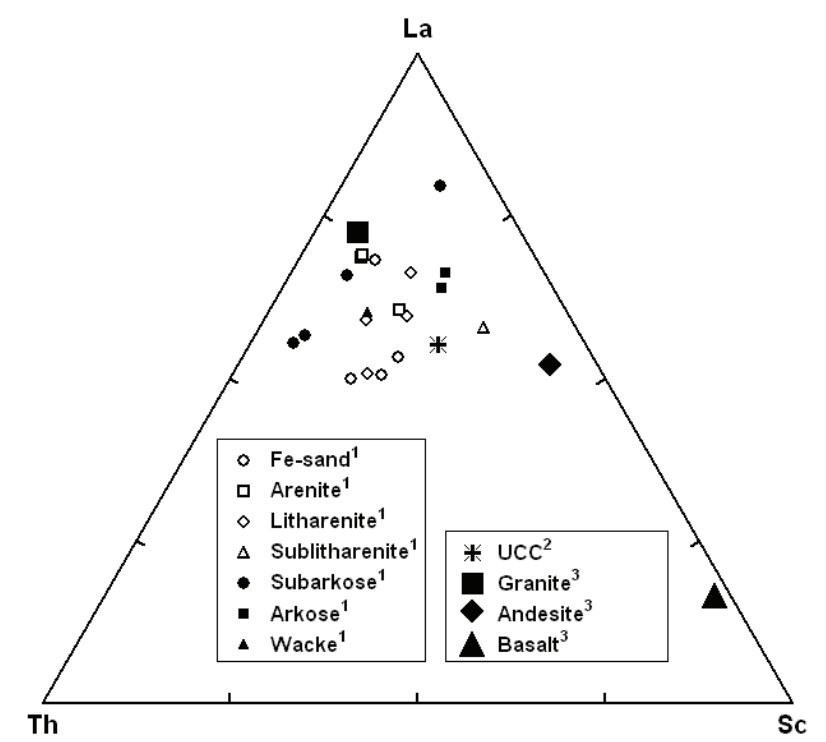

Fig.9. La-Th-Sc ternary diagram for the Sillakkudi sandstones. ${ }^{1}$ This study; ${ }^{2}$ Taylor and McLennan (1985); ${ }^{3}$ Condie (1993). 
Table 3. Range of elemental ratios of Sillakkudi sandstones in this study compared to the ratios in similar fractions derived from felsic rocks, mafic rocks and upper continental crust (UCC)

\begin{tabular}{lcccc}
\hline $\begin{array}{l}\text { Elemental } \\
\text { Ratio }\end{array}$ & $\begin{array}{c}\text { Range of sandstones from } \\
\text { Sillakkudi Formation } \\
(n=20)\end{array}$ & $\begin{array}{c}\text { Range of sediment } \\
\text { from felsic sources }\end{array}$ & $\begin{array}{c}\text { Range of sediment } \\
\text { from mafic sources }^{2}\end{array}$ & $\begin{array}{c}\text { Upper continental } \\
\text { crust }^{3}\end{array}$ \\
\hline $\mathrm{Eu} / \mathrm{Eu}{ }^{*}$ & $0.35-1.73$ & $0.40-0.94$ & $0.71-0.95$ & 0.63 \\
$(\mathrm{La} / \mathrm{Lu})_{\mathrm{cn}}$ & $4.10-23.53$ & $3.00-27.00$ & $1.10-7.00$ & 9.73 \\
$\mathrm{La} / \mathrm{Sc}$ & $1.93-9.36$ & $2.5-16.3$ & $0.43-0.86$ & 2.21 \\
$\mathrm{Th} / \mathrm{Sc}$ & $0.41-6.57$ & $0.84-20.5$ & $0.05-0.22$ & 0.79 \\
$\mathrm{Th} / \mathrm{Co}$ & $0.14-5.01$ & $0.67-19.4$ & $0.04-1.4$ & 0.63 \\
$\mathrm{Th} / \mathrm{Cr}$ & $0.23-2.94$ & $0.13-2.7$ & $0.018-0.046$ & 0.13 \\
$\mathrm{Cr} / \mathrm{Th}$ & $0.34-4.28$ & $4.00-15.0$ & $25-500$ & 7.76 \\
$\mathrm{La} / \mathrm{Co}$ & $1.23-9.29$ & $1.80-13.8$ & $0.14-0.38$ & 1.76 \\
\hline
\end{tabular}

${ }^{1}$ This study; ${ }^{2}$ Cullers (1994), (2000); Cullers and Podkovyrov (2000); Cullers et al. (1988); Armstrong-Altrin (2009); ${ }^{3}$ McLennan (2001); Taylor and McLennan (1985)

sub-litharenite and litharenite plot between average granite, and UCC compositions, which suggests that the Sillakkudi sandstones were derived probably by the influence of felsic source rocks.

Similarly, ratios such as Eu/Eu* ${ }^{*} \mathrm{La} / \mathrm{Sc}, \mathrm{Th} / \mathrm{Sc}, \mathrm{Th} / \mathrm{Co}$, and $\mathrm{Th} / \mathrm{Cr}$ are significantly different in felsic and mafic rocks and may allow constraints on the average provenance composition (Cullers, 1995, 2000; Cox et al. 1995). Th/Sc, $\mathrm{Th} / \mathrm{Co}, \mathrm{Th} / \mathrm{Cr}, \mathrm{Cr} / \mathrm{Th}$, and $\mathrm{La} / \mathrm{Sc}$ ratios of Sillakkudi sandstones are compared with those of sediments derived from felsic and mafic rocks as well as to UCC values (Table 3). In this comparison the Sillakkudi sandstones fall within the range of felsic sediments and supports again for the felsic source rocks.

The REE pattern and Eu anomaly in the sedimentary rocks will provide important clues for the source rock characteristics (Taylor and McLennan, 1985). Higher LREE/ HREE ratios and negative Eu anomaly are generally found in felsic rocks, whereas the mafic rocks exhibit low LREE/ HREE ratio and low negative Eu anomaly (Cullers, 1994). The positive Eu anomaly was generally found in Proterozoic rocks (TTG), granodiorite and quartz diorite. The low positive Eu anomaly in TTG is resulted from hornblende melt equilibria (Cullers and Graf, 1984). In this study, the Chondrite normalized REE plots (Fig. 4) display fractionated REE patterns $\left(\mathrm{La}_{\mathrm{CN}} / \mathrm{Yb}_{\mathrm{CN}}=\sim 3.21-19.90\right)$, which are characterized by relatively flat $\operatorname{HREE}\left(\mathrm{Gd}_{\mathrm{CN}} / \mathrm{Yb}_{\mathrm{CN}}=0.73-\right.$ 2.57), enriched LREE $\left(\mathrm{La} / \mathrm{Sm}_{\mathrm{CN}}=3.39-5.82\right)$ and negative $\mathrm{Eu}$ anomaly $\left(\mathrm{Eu} / \mathrm{Eu}^{*}=0.80 \pm 0.03\right)$ similar to UCC. In addition, the Sillakkudi sandstones show enriched LREE/ HREE ratio (4.30-13.66; 9.11 $\pm 2.62 ; \mathrm{n}=20)$, which suggests that these sandstones were mainly derived from the felsic source rocks (Taylor and McLennan, 1985).

To know the probable source rocks for the Sillakkudi sandstones (Fig. 10) the average REE patterns are compared with those of charnockites $(n=21)$ and gneisses $(n=23)$ belongs to Kerala Khondalite Belt (Allen et al., 1985; Chacko et al., 1992), and granite $(n=6)$ and gneisses $(n=$ 9) from Dharwar Craton (Jayananda et al. 1995; Stähle et al. 1987). These areas belong relatively close to the study area and are supposed to contribute sediments to the study area. The chondrite normalized REE patterns of Sillakkudi sandstones show LREE enriched, flat HREE and with significant negative Eu anomaly. However, considering the SREE (especially HREE) content and the size of the negative $\mathrm{Eu}$ anomaly, these sandstones are very similar to the gneisses of Dharwar Craton. This implies that the Sillakkudi sandstones received a higher contribution of sediments from Dharwar craton than Kerala khondalite belt.

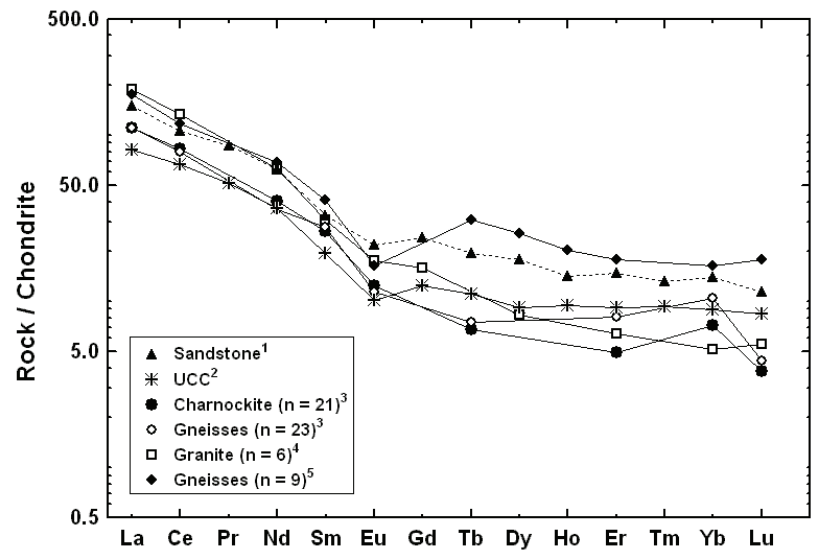

Fig.10. Chondrite-normalized REE patterns. $n=$ number of samples; ${ }^{1}$ This study (Sillakkudi sandstones); ${ }^{2}$ average upper continental crust (UCC; Taylor and McLennan, 1985); ${ }^{3}$ Kerala Khondalite Belt (Allen et al. 1985; Chacko et al. 1992); ${ }^{4}$ Dharwar Craton (Jayananda et al. 1995); ${ }^{5}$ Dharwar Craton (Stähle et al. 1987). 


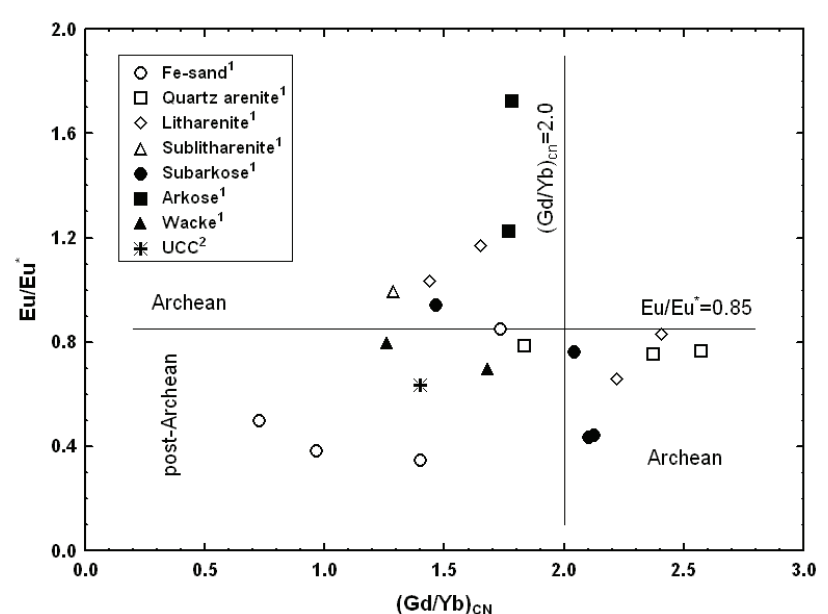

Fig.11. Plot of $\mathrm{Eu} / \mathrm{Eu}^{*}$ versus $(\mathrm{Gd} / \mathrm{Yb})_{\mathrm{CN}}$ for the Sillakkudi sandstones. Fields after McLennan and Taylor (1991). ${ }^{1}$ This study; ${ }^{2}$ Taylor and McLennan (1985).

The granitic rocks formed during Phanerozoic Eon have more K-feldspar rich granites and thereby reflect less depletion of $\mathrm{Eu}$, and high depletion of HREE with $\mathrm{Gd}_{\mathrm{CN}} /$ $\mathrm{Yb}_{\mathrm{CN}}$ ratio < 2 (McLennan 1989; Taylor and McLennan 1985). In the $\mathrm{Eu} / \mathrm{Eu}^{*}{ }^{*} \mathrm{vs}_{\mathrm{CN}} / \mathrm{Yb}_{\mathrm{CN}}$ plot (Fig.11) the fe-sand and wacke types plot in the Phanerozoic field (K-feldspar rich granites and there by reflect less depletion of Eu), which shows $\mathrm{Gd}_{\mathrm{CN}} / \mathrm{Yb}_{\mathrm{CN}}$ ratio $<2.0$, whereas arkose and litharenites show $\mathrm{Gd}_{\mathrm{N}} / \mathrm{Yb}_{\mathrm{N}}$ ratio more than 2 and low $\mathrm{Eu} / \mathrm{Eu}^{*}$ ratio. However, some arkose, sub-arkoses, and litharenite types show enriched $\mathrm{Eu} / \mathrm{Eu}^{*}$ and $\mathrm{Gd}_{\mathrm{CN}} / \mathrm{Yb}_{\mathrm{CN}}$ ratio $<2.0$, which suggests the Archaean igneous source.

\section{CONCLUSIONS}

The Sillakkudi sandstones are classified as fe-sand, quartz arenite, litharenite, sub-arkose, arkose, wacke and sub-litharenite based on major element geochemistry. Lower concentrations of immobile trace elements like $\mathrm{Cr}, \mathrm{Co}, \mathrm{V}$, and Sc suggest the felsic source rock provenance, which is also supported by the $\mathrm{Th} / \mathrm{Sc}, \mathrm{Th} / \mathrm{Co}, \mathrm{Th} / \mathrm{Cr}, \mathrm{Cr} / \mathrm{Th}$, and $\mathrm{La} / \mathrm{Sc}$ ratios. Similarly, the La-Th-Sc ternary and $\mathrm{La} / \mathrm{Sc}$ vs Th/Co plots also reveal the felsic character of the source rocks. Enrichment of $\mathrm{Zr}$ identified from $\mathrm{Th} / \mathrm{Sc}$ versus $\mathrm{Zr} / \mathrm{Sc}$ plot in fe-sands and sub-arkose types suggests the influence of sedimentary processes such as sediment recycling and sorting. However, the zircon geochemistry did not affect the REE distribution and its patterns in the Sillakkudi sandstones. The comparison of REE patterns and its Eu anomalies to the source rocks reveals that the Sillakkudi sandstones received a higher contribution of sediments from Dharwar Craton than Kerala Khondalite Belt. We conclude that the REE patterns and $\mathrm{Eu}$ anomalies are well preserved in the Sillakkudi sandstones and are highly reliable indicator of source rocks, even though the geochemical composition can be affected by processes such as hydraulic sorting during transportation.

Acknowledgements: We are thankful to Dr. J. Madhavaraju and Dr. S. Srinivasalu for their valuable suggestions and constant encouragement during this study. R. Nagendra is thankful to the UGC, New Delhi for the financial support. We would like to thank Director, NGRI, Dr. Balaram (Head, Geochemical laboratory), Sathyanarayanan (NGRI), and Gnaneshwar Rao (NGRI), for their help in geochemical analysis. We are also grateful to the anonymous reviewers for helpful comments to improve our paper.

\section{References}

Allen, P., Condie, K.C. and Narayana, B.L. (1985) Geochemistry of prograde and retrograde charnockite-gneiss reactions in southern India. Geochim. Cosmochim. Acta, v.49, pp.323336.

Araújo, C.E.G., Pinéo, T.R.G., Caby, R., Costa, F.G., Cavalcante, J.C., Vasconcelos, A.M. and Rodrigues, J.B. (2010) Provenance of the Novo Oriente Group, southwestern Ceará Central Domain, Borborema Province (NE-Brazil): A dismembered segment of a magma-poor passive margin or a restricted rift-related basin? Gondwana Res., v.18, pp.497513.

Armstrong-Altrin, J.S. (2009) Provenance of sands from Cazones, Acapulco, and Bahía Kino beaches, Mexico, Revista Mexicana de Ciencias Geológicas, v.26(3), pp.764-782.

Armstrong-Altrin, J.S. and Verma, S.P. (2005) Critical evaluation of six tectonic setting discrimination diagrams using geo- chemical data of Neogene sediments from known tectonic setting. Sediment. Geol., v.177, pp.115-129.

Armstrong-Altrin, J.S., Lee, Y.I., Verma, S.P. and Ramasamy, S. (2004) Geochemistry of sandstones from the upper Miocene Kudankulam formation, Southern India: Implications for provenance, weathering, and tectonic setting. Jour. Sed. Res., v.74(2), pp.285-297.

Asiedu., D. K., Suzuki, S., Nogami, K. and Shibata, T. (2000) Geochemistry of Lower Cretaceous sediments, Inner Zone of Southwest Japan: Constraints on provenance and tectonic environment. Geochem. Jour., v.34, pp.155-173.

Ayyasami, K. (2006) Role of oysters in biostratigraphy: A case study from the Cretaceous of the Ariyalur area, southern India. Geosci. Jour., v.10(3), pp.237-247.

BANERJI, R.K. (1979) On the occurrence of Tertiary algal reefs in the Cauvery basin and their stratigraphic relationship. Geol. 
Surv. India Misc. Publ., v.45, pp.181-196.

Bhatia, M.R. (1983) Plate tectonics and geochemical composition of sandstones. Jour. Geol., v.91, pp.611-627.

BнAтіA, M.R. (1985) Rare earth element geochemistry of Australian Paleozoic greywackes and mudstones: provenance tectonic control. Sediment. Geol., v.45, pp.97-113.

BlanFord, H.F. (1862) On the Cretaceous and other rocks of the South Arcot and Trichinopoly districts. Mem. Geol. Soc. India., v.4, pp.1-217.

Blatt, H. G., Middleton, G. V. and Murray, R. C. (1980) Origin of Sedimentary Rocks. 2nd ed., Prentice-Hall, New Jersey, $634 \mathrm{p}$.

Bock, B., McLennan, S.M. and Hanson, G.N. (1998) Geochemistry and provenance of the Middle Ordovician Austin Glen Member (Normanskill Formation) and the Taconian Orogeny in New England. Sedimentol., v.45, pp.635-655.

Carranza-Edwards, A., Kasper-Zubillaga, J.J., Rosales-Hoz, L., Alfredo-Morales, E. and Santa-Cruz, R.L. (2009) Beach sand composition and provenance in a sector of the southwestern Mexican Pacific. Revista Mexicana de Ciencias Geológicas, v.26(2), pp.433-447.

Chacko, T., Ravindra Kumar G.R., Meen, J.K. and Rogers, J.W. (1992) Geochemistry of high-grade supracrustal rocks from the Kerala Khondalite Belt and adjacent massif charnockites, South India. Precambrian Res., v.55, pp.469-489

Chakrabarti, R., Basu, A.R. and Chakrabarti, A. (2007) Trace element and $\mathrm{Nd}$-isotopic evidence for sediment sources in the mid-Proterozoic Vindhyan Basin, Central India. Precambrian Res., v.159, pp.260-274.

Chakrabarti, G., Shome, D., Bauluz, B. and Sinha, S. (2009) Provenance and Weathering History of Mesoproterozoic Clastic Sedimentary Rocks from the Basal Gulcheru Formation, Cuddapah Basin, India. Jour. Geol. Soc. India, v.74, pp.119-130.

Condie, K.C. (1991) Another look at rare earth elements in shales. Geochim. Cosmochim. Acta., v.55, pp.2527-2531.

Condie, K.C. (1993) Chemical composition and evolution of the upper continental crust: Contrasting results from surface samples and shales. Chem. Geol., v.104, pp.1-37.

Condie, K.C., LeE, D. and FARMER, L. (2001) Tectonic setting and provenance of the Neoproterozoic Uinta Mountain and Big Cootonwood groups, northern Utah: constraints from geochemistry, Nd isotopes, and detrital modes. Sediment. Geol., v.141-142, pp.443-464.

Cox, R., Low, D.R. and Cullers, R.L., (1995) The influence of sediment recycling and basement composition on evolution of mudrock chemistry in the southwestern United States. Geochim. Cosmochim. Acta., v.59(14), pp.2919-2940.

Cullers, R. L. (1994) The controls on the major and trace element variation of shales, siltstones and sandstones of Pennsylvanian - Permian age from uplifted continental blocks in Colorado to platform sediment in Kansas, USA. Geochim. Cosmochim. Acta., v.58(22), pp.4955-4972.

Cullers, R.L. (1995) The controls on the major and trace element evolution of shales, siltstones and sandstones of Ordovician to Tertiary age in the Wet Mountain region, Colorado, U.S.A. Chem. Geol., v.123(1-4), pp.107-131.
Cullers, R.L. (2000) The geochemistry of shales, siltstones and sandstones of Pennsylvanian-Permian age, Colorado, U.S.A.: implications for provenance and metamorphic studies. Lithos, v.51, pp.305-327.

Cullers, R.L. (2002) Implications of elemental concentrations for provenance, redox conditions, and metamorphic studies of shales and limestones near Pueblo, CO, USA. Chem. Geol., v.191(4), pp.305-327.

Cullers, R.L. and Graf, J. (1984) Rare earth element in igneous rocks of the continental crust: intermediate and silicic rocks, ore petrogenesis. In: P. Henderson (Ed.), Rare Earth Geochemistry. Elsevier, pp.275-316.

Cullers, R.L. and Podkovyrov, V.N. (2000) Geochemistry of the Mesoproterozoic Lakhanda shales in southeastern Yakutia, Russia: implications for mineralogical and provenance control, and recycling. Precambrian Res., v.104(1-2), pp.77-93.

Cullers, R.L., Basu, A. and Suttner, L. (1988) Geochemical signature of provenance in sand-size material in soils and stream sediments near the Tobacco Root batholith, Montana, USA. Chem. Geol., v.70(4), pp.335-348.

Das, B.K., Al-Mikhlafi, A.S. and Kaur, P. (2006) Geochemistry of Mansar lake sediments, Jammu, India: Implication for source-area weathering, provenance, and tectonic setting. Jour. Asian Earth Sci., v.26(6), pp.649-668.

Dey, S., Rai, A.K. and Chaki, A. (2009) Palaeoweathering, composition and tectonics of provenance of the Proterozoic intracratonic Kaladgi-Badami basin, Karnataka, southern India: Evidence from sandstone petrography and geochemistry. Jour. Asian Earth Sci, v.34, pp.703-715.

DiCKINSON, W.R. (1970) Interpreting detrital modes of greywacke and arkose. Jour. Sediment. Petrol., v.40, pp.695-707.

FANTI, F. (2009). Bentonite chemical features as proxy of late Cretaceous provenance changes: A case study from the Western Interior Basin of Canada. Sediment. Geol., v.217, pp.112127.

Feng, R. and KerRich, R. (1990) Geochemistry of fine-grained clastic sediments in the Archean Abitibi greenstones belt, Canada: implications for provenance and tectonic setting. Geochim. Cosmochim. Acta., v.54, pp.1061-1081.

Garcia, D., Ravenne, C., Maréchal, B. and Moutte, J. (2004) Geochemical variability induced by entrainment sorting: quantified signals for provenance analysis. Sediment. Geol., v.171(1-4), pp.113-128.

GAzzI, P. (1966) Le arenarie del flysch sopracretaceo dell'Appennino modensese: Correlazioni con il flysch di Monghidoro. Miner. Petrographica Acta, v.12, pp.69-97.

Govindan, A., Ravindran, C.N. and Rangaraju, M.K. (1996) Cretaceous stratigraphy and planktonic foraminiferal zonation of Cauvery Basin, South India. In: A. Sahni (Ed.), Cretaceous Stratigraphy and Palaeoenvironments. Mem. Geol. Soc. India, no.37, pp.155-187.

Herron, M.M. (1988) Geochemical classification of terrigenous sands and shales from core or log data. Jour. Sediment. Petrol., v.58, pp.820-829.

Hurowitz, J.A. and McLennan, S.M. (2005) Geochemistry of Cambro-Ordovician Sedimentary Rocks of the Northeastern United States: Changes in Sediment Sources at the Onset of 
Taconian Orogenesis. Jour. Geol., v.113, pp.571-587.

Jayananda, M., Martin, H., Peucat, J.J. and Mahabaleswar, B. (1995) Late Archaean crust-mantle interactions: geochemistry of LREE-enriched mantle derived magmas. Example of the Clospet batholith, southern India. Contrib. Mineral. Petrol., v.119(2-3), pp.314-329.

Joo, Y.J., Lee, Y.I. and BAI, Z. (2005) Provenance of the Qingshuijian Formation (Late Carboniferous), NE China: Implications for tectonic processes in the northern margin of the North China block. Sediment. Geol., v.177, pp.97- 114.

Kasanzu, C., Maвoкo, M.A.H. and Manya, S. (2008) Geochemistry of fine grained clastic sedimentary rocks of the Neoproterozoic Ikorongo Group, NE Tanzania: Implications for provenance and source rock weathering. Precambrian Res., pp.201-213.

Kasper-Zubillaga, J. J., Carranza-Edwards, A. and MortonBermea, O. (2008) Heavy Minerals and Rare Earth Elements in Coastal and Inland Dune Sands of El Vizcaino Desert, Baja California Peninsula, Mexico. Marine Georesources \& Geotechnology, v.26(3), pp.172-188.

Khudoley, A.K., Rainbird, R.H., Stern, R.A., Kropachev, A.P., Heaman, L.M., Zanin, A.M., Podkovyrov, V.N., Belova, V.N. and SukHorukov, V.I. (2001) Sedimentary evolution of the Riphean-Vendian basin of southeastern Siberia. Precambrian Res., v.111, pp.129-163.

Kimberley, M.M. and Grandstaff, D.F. (1986) Profiles of elemental concentrations in Precambrian paleosols on basaltic and granitic parent materials. Precambrian Res., v.32, pp.133154.

LAmaskin, T.A., Dorsey, R. and Vervoort, J.D. (2008) Tectonic controls on mudrock geochemistry, Mesozoic rocks of eatern oregon and western Idaho, U.S.A.: Implications for Cordilleran tectonics. Jour. Sediment. Res., v.78, pp.765-783.

Lindsey, D.A. (1999) An evaluation of alternative chemical classifications of sandstones. USGS Open File Report 99-34, $23 \mathrm{p}$.

Liu, S., Lin, G., Liu, Y., Zhou, Y., Gong, F. and Yan, Y. (2007) Geochemistry of Middle Oligocene-Pliocene sandstones from the Nanpu Sag, Bohai Bay Basin (Eastern China): Implications for provenance, weathering, and tectonic setting. Geochem. Jour., v.41(5), pp.359-378.

Madhavaraju, J. (1996) Petrofacies, geochemistry and depositional environments of Ariyalur Group of sediments, Tiruchirapalli Cretaceous, Tamil Nadu. Ph.D. thesis, University of Madras, 160 .

Madhavaraju, J. and Ramasamy, S. (1999a) Rare earth elements in limestones of Kallankurichchi Formation of Ariyalur Group, Tiruchirapalli Cretaceous, Tamil Nadu. Jour. Geol. Soc. India, v.54, pp.291-301.

Madhavaraju, J. and Ramasamy, S. (1999b) Microtextures on quartz grains of Campanian - Maastrichtian sediments of Ariyalur Group of Tiruchirapalli Cretaceous, Tamil Nadu Implication on depositional environments. Jour. Geol. Soc. India, v.54, pp.647-658.

Madhavaraju, J. and Ramasamy, S. (2001) Clay mineral assemblages and rare earth element distribution in the sediments of Ariyalur Group, Tiruchirapalli District, Tamil
Nadu -Implication for paleoclimate. Jour. Geol. Soc. India, v.58, pp.69-77.

Madhavaraju, J. and Lee, Y.I. (2009) Geochemistry of the Dalmiapuram Formation of the Uttatur Group (Early Cretaceous), Cauvery basin, southeastern India: Implications on provenance and paleo-redox conditions. Revista Mexicana de Ciencias Geológicas, v.26(2), pp.380-394.

Madhavaraju, J., Ramasamy, S., Ruffell. A. and Mohan, S.P. (2002) Clay mineralogy of the Late Cretaceous and Early Tertiary Successions of the Cauvery Basin (southeastern India): Implications for sediment source and palaeoclimates at the K/ T boundary. Cretaceous Res., v.23, pp.153-163.

Maravelis, A. and Zelilidis, A. (2009) Petrography and geochemistry of the late Eocene-early Oligocene submarine fans and shelf deposits on Lemnos Island, NE Greece. Implications for provenance and tectonic setting. Geol. Jour. DOI: $10.1002 /$ gj.1183.

McLennan, S.M. (1989) Rare earth elements in sedimentary rocks; influence of provenance and sedimentary processes, in: B.R. Lipin, G.A. McKay (Eds.), Geochemistry and Mineralogy of Rare Earth Elements, Rev. Mineral., v.21, pp.169-200.

McLennan, S.M. and TAYLOR, S.R. (1991) Sedimentary rocks and crustal evolution: tectonic setting and secular trends. Jour. Geol, v.99, pp.1-21.

McLennan, S.M., Nance, W.B. and Taylor, S.R. (1980) Rare earth element-Thorium correlation in sedimentary rocks, and the composition of the continental crust. Geochim. Cosmochim. Acta., v.44, pp.1833-1839.

McLennan, S.M., Taylor, S.R. and Eriksson, K.A. (1983) Geochemistry of Archaean shales from the Pilbara Supergroup, Western Australia. Geochim. Cosmochim. Acta., v.47(7), pp.1211-1222.

McLennan, S.M., TaYlor, S.R., Mcculloch, M.T. and Maynard, J.B. (1990) Geochemical and Nd-Sr isotopic composition of deep sea turbidites: Crustal evolution and plate tectonic associations. Geochim. Cosmochim. Acta., v.54, pp.2015-2050.

McLennan, S.M., Hemming, S., Mcdaniel, D.K. and Hanson, G.N. (1993) Geochemical approaches to sedimentation, provenance, and tectonics. In: M.J. Johnson and A. Basu (Eds.), Processes Controlling the Composition of Clastic Sediments. Geol. Soc. Amer. Spec. Paper, v.284, pp.21-40.

Nagarajan, R., Armstrong-Altrin, J.S., Nagendra, R., Madhavaraju, J. and Moutte, J. (2007a) Petrography and geochemistry of terrigenous sedimentary rocks in the Neoproterozoic Rabanpalli Formation, Bhima Basin, southern India: Implications for paleoweathering condition, provenance, and source rock composition. Jour. Geol. Soc. India, v.70(2), pp.297-312.

Nagarajan, R., Madhavaraju, J., Nagendra, R., ArmstrongAltrin, J.S. and Moutte, J. (2007b) Geochemistry of Neoproterozoic shales of Rabanpalli Formation, Bhima Basin, Northern Karnataka, Southern India: Implications for provenance and paleoredox conditions. Revista Mexicana Ciencias Geológicas, v.24(2), pp.150-160.

Nesbitt, H.W. and Young, Y.M. (1982) Early Proterozoic climates and plate motions inferred from major element chemistry of lutites. Nature, v.299, pp.715-717. 
Nesbitt, H.W. and Young, Y.M. (1984) Prediction of some weathering trends of plutonic and volcanic rocks based on thermodynamic and kinetic considerations. Geochim. Cosmochim. Acta., v.48, pp.1523-1534.

Pe-Piper, G., Triantafyllidis, S. and Piper, D.J.W. (2008) Geochemical identification of clastic sediment provenance from known sources of similar geology: the Cretaceous Scotian Basin, Canada. Jour. Sediment. Res, v.78, pp.595-607.

Pettijohn, F.H., Potter, P.E. and Siever, R. (1972) Sand and Sandstone. Springer-Verlag, New York, 618p.

Prabhakar, K.N. and Zutshi, P.L. (1993) Evolution of southern part of Indian east coast basins. Jour. Geol. Soc. India, v.41, pp.215-230.

Ramanathan, S. (1979) Tertiary formations of south India. Geol. Surv. India Misc. Publ., v.45, pp.165-180.

Ramasamy, S. and BANERJ, R.K. (1991) Geology, petrography and stratigraphy of pre-Ariyalur sequence in Tiruchirapalli District, Tamil Nadu. Jour. Geol. Soc. India, v.37, pp.577-594.

Ramasamy, S., Madhavaraju, J. and Banerji, R.K. (1995) Paleoenvironmental indicators of the pre-Ariyalur sequence in Tiruchirapalli District, Tamil Nadu, India (abstract), In 2nd South Asia Geological Congress, Colombo, Sri Lanka, pp.158159.

Ranjan, N. and BanerJee, D.M. (2009) Central Himalayan crystallines as the primary source for the sandstone-mudstone suites of the Siwalik Group: New geochemical Evidence, Gondwana Res., v.16, pp.687-696.

Rangaraju, M.K., Agarwal. A. and Prabhakar, K.N. (1993) Tectono-stratigraphy, structural styles, evolutionary model and hydrocarbon habitat, Cauvery and Palar basins, In: S.K. Biswas, A. Dave, P. Garg, J. Pandey, A. Maithani and N.J. Thomas (Eds.), Proc. Second Seminar on Petroliferous Basins of India: Dehra Dun, Indian Petroleum Publ., v.1, pp.371388.

Roddaz, M., Viers, J., Brusset, S., Baby, P., Boucayrand, C. and HÉrAIL, G. (2006) Controls on weathering and provenance in the Amazonian foreland basin: Insights from major and trace element geochemistry of Neogene Amazonian sediments. Chem. Geol., v.226, pp.31- 65.

Roser, B.P. and Korsch, R.J. (1986) Determination of tectonic setting of sandstone-mudstone suites using $\mathrm{SiO}_{2}$ content and $\mathrm{K}_{2} \mathrm{O} / \mathrm{Na}_{2} \mathrm{O}$ ratio. Jour. Geol., v.94(5), pp.635-650.

Ryan, K.M. and Williams, D.M. (2007) Testing the reliability of discrimination diagram for determining the tectonic depositional environment of ancient sedimentary basins. Chem. Geol., v.242, pp.103-125.

Sastry, M.V.A., Mamgain, V.D. and RaO, B.R. (1972) Ostracod fauna of the Ariyalur Group (Upper Cretaceous) Tiruchirapalli District, Tamil Nadu. Part I. Lithostratigraphy of the Ariyalur Group. Mem. Geol. Surv. India, Palaentologica Indica, New Series, 40, pp.1-48.

Selvaraj, K. and Chen, C.-T.A. (2006) Moderate chemical weathering of subtropical Taiwan: Constraints from solid-phase geochemistry of sediments and sedimentary rocks. The Journal of Geology, v.114(1), pp.101-116.

Sinha, S., Islam, R., Ghosh, S.K., Rohtash Kumar and Sangode,
S.J. (2007) Geochemistry of Neogene Siwalik mudstones along Punjab re-entrant, India: Implications for source area weathering, provenance and tectonic setting. Curr. Sci., v.92(8), pp.1103-1113.

Sharma, A. and RAJAmANI, V. (2001) Weathering of charnockites and sediment production in the catchment area of the Cauvery River, south India. Sediment. Geol., v.143, pp.169-184.

StäHle, H.J., Raith, M., Hoernes, S. and Delfs, A. (1987) Element mobility during incipient granulite formation at Kabbaldurga, southern India. Jour. Petrol., v.28, pp.803-834.

Sundaram, R. and RaO, P.S. (1986) Lithostratigraphy of the Upper Cretaceous rocks in the Vridhachalam area, south Arcot district, Tamil Nadu, South India. Geol, Surv. India Spec. Publ., v.11, pp.515-522.

Sundaram, R., Henderson, R.A., Ayyasami, K. and Stilwell, J.D. (2001) A lithostratigraphic revision and palaeoenvironmental assessment of the Cretaceous system exposed in the onshore Cauvery Basin, southern India. Cretaceous Res., v.22, pp.743762.

TAYLOR, S.R. and McLennan, S.M. (1985) The Continental Crust: Its Composition and Evolution. London, Blackwell Scientific Publications, 312p.

Umazano, A. M., Bellosi, E. S., Visconti, G., Jalfin, A.G. and Melchor, R. N. (2009) Sedimentary record of a Late Cretaceous volcanic arc in central Patagonia: petrography, geochemistry and provenance of fluvial volcaniclastic deposits of the Bajo Barreal Formation, San Jorge Basin, Argentina. Cretaceous Res., v.30, pp.749-766.

Van De Kamp, P.C. and Leake, B.E. (1995) Petrology and geochemistry of siliciclastic rocks of mixed feldspathic and ophiolitic provenance in the Northern Apennines, Italy. Chem. Geol., v.122, pp.1-20.

Van Staden, A., Naidoo, T., Zimmermann, U. and Germs, G.J.B. (2006) Provenance analysis of selected clastic rocks in Neoproterozoic to lower Paleozoic successions of southern Africa from the Gariep Belt and the Kango Inlier. South African Jour. Geol., v.109, pp.215-232

Varga, A., Raucsik, B., Hartyáni, Z. and Szakmány, G. (2007) Paleoweathering conditions of Upper Carboniferous siliciclastic rocks of SW Hungary. Central European Geology, v.50(1), pp.3-18.

Wanas, H.A. and Abdel-Maguid, N.M. (2006) Petrography and geochemistry of the Cambro-Ordovician Wajid Sandstone, southwest Saudi Arabia: Implications for provenance and tectonic setting. Jour. Asian Earth Sci., v.27(4). pp. 416-429.

Wani, H. and Mondal, M.E.A. (2010) Petrological and geochemical evidence of the Paleoproterozoic and the MesoNeoproterozoic sedimentary rocks of the Bastar craton, Indian Peninsula: Implications on paleoweathering and Proterozoic crustal evolution. Jour. Asian Earth Sci., v.38(5), pp.220232.

Zimmermann, U. and Spalletti, L. S. (2009) Provenance of the Lower Paleozoic Belcarce Formation (Tandilia System, Buenos Aires Province, Argentina): Implications for paleogeographic reconstructions of SW Gondwana. Sediment. Geol., v.219, pp.7-23.

(Received: 18 December 2009; Revised form accepted: 19 May 2010) 